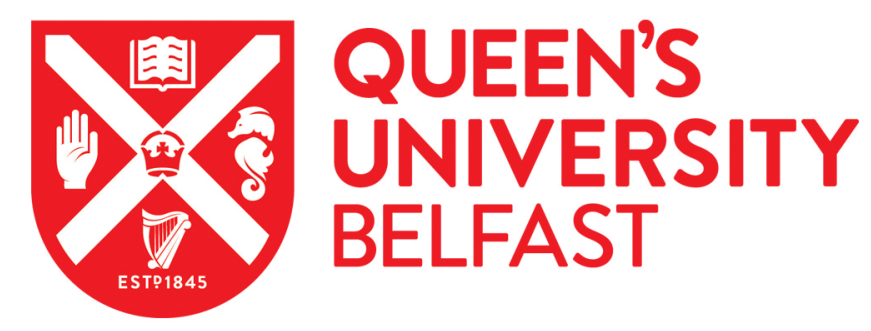

\title{
Constraints on explosive silicon burning in core-collapse supernovae from measured $\mathrm{Ni} / \mathrm{Fe}$ ratios
}

Jerkstrand, A., Timmes, F. X., Magkotsios, G., Sim, S. A., Fransson, C., Spyromilio, J., Müller, B., Heger, A., Sollerman, J., \& Smartt, S. J. (2015). Constraints on explosive silicon burning in core-collapse supernovae from measured Ni/Fe ratios. The Astrophysical Journal, 807(1), [110]. https://doi.org/10.1088/0004-637X/807/1/110

\section{Published in:}

The Astrophysical Journal

Document Version:

Publisher's PDF, also known as Version of record

Queen's University Belfast - Research Portal:

Link to publication record in Queen's University Belfast Research Portal

Publisher rights

(c) 2015. The American Astronomical Society. All rights reserved.

\section{General rights}

Copyright for the publications made accessible via the Queen's University Belfast Research Portal is retained by the author(s) and / or other copyright owners and it is a condition of accessing these publications that users recognise and abide by the legal requirements associated with these rights.

Take down policy

The Research Portal is Queen's institutional repository that provides access to Queen's research output. Every effort has been made to ensure that content in the Research Portal does not infringe any person's rights, or applicable UK laws. If you discover content in the Research Portal that you believe breaches copyright or violates any law, please contact openaccess@qub.ac.uk. 


\title{
CONSTRAINTS ON EXPLOSIVE SILICON BURNING IN CORE-COLLAPSE SUPERNOVAE FROM MEASURED Ni/Fe RATIOS
}

\author{
A. Jerkstrand ${ }^{1}$, F. X. Timmes ${ }^{2,3}$, G. Magkotsios ${ }^{3}$, S. A. Sim $^{1}$, C. Fransson ${ }^{4}$, J. Spyromilio ${ }^{5}$, B. Müller ${ }^{6}$, A. Heger ${ }^{6,7,8}$, \\ J. Sollerman ${ }^{4}$, AND S. J. SMarti ${ }^{1}$ \\ ${ }^{1}$ Astrophysics Research Centre, School of Mathematics and Physics, Queen's University Belfast, Belfast BT7 1NN, UK; a.jerkstrand@qub.ac.uk \\ ${ }^{2}$ School of Earth and Space Exploration, Arizona State University, Tempe, AZ, USA \\ ${ }^{3}$ Joint Institute for Nuclear Astrophysics, Notre Dame, IN 46556, USA \\ ${ }^{4}$ The Oskar Klein Centre, Department of Astronomy, Stockholm University, Albanova, SE-10691 Stockholm, Sweden \\ ${ }^{5}$ ESO, Karl-Schwarzschild-Strasse 2, D-85748 Garching, Germany \\ ${ }^{6}$ Monash Centre for Astrophysics, School of Physics and Astronomy, Monash University, VIC 3800, Australia \\ ${ }^{7}$ School of Physics and Astronomy, University of Minnesota, Minneapolis, MN 55455, USA \\ ${ }^{8}$ Department of Physics and Astronomy, Shanghai Jiao-Tong University, Shanghai 200240, China \\ Received 2015 March 6; accepted 2015 May 19; published 2015 July 6
}

\begin{abstract}
Measurements of explosive nucleosynthesis yields in core-collapse supernovae provide tests for explosion models. We investigate constraints on explosive conditions derivable from measured amounts of nickel and iron after radioactive decays using nucleosynthesis networks with parameterized thermodynamic trajectories. The $\mathrm{Ni} / \mathrm{Fe}$ ratio is for most regimes dominated by the production ratio of ${ }^{58} \mathrm{Ni} /\left({ }^{54} \mathrm{Fe}+{ }^{56} \mathrm{Ni}\right)$, which tends to grow with higher neutron excess and with higher entropy. For SN 2012ec, a supernova (SN) that produced a Ni/Fe ratio of $3.4 \pm 1.2$ times solar, we find that burning of a fuel with neutron excess $\eta \approx 6 \times 10^{-3}$ is required. Unless the progenitor metallicity is over five times solar, the only layer in the progenitor with such a neutron excess is the silicon shell. SNe producing large amounts of stable nickel thus suggest that this deep-lying layer can be, at least partially, ejected in the explosion. We find that common spherically symmetric models of $M_{\text {ZAMS }} \lesssim 13 M_{\odot}$ stars exploding with a delay time of less than one second $\left(M_{\text {cut }}<1.5 M_{\odot}\right)$ are able to achieve such silicon-shell ejection. SNe that produce solar or subsolar Ni/Fe ratios, such as SN 1987A, must instead have burnt and ejected only oxygen-shell material, which allows a lower limit to the mass cut to be set. Finally, we find that the extreme Ni/Fe value of 60-75 times solar derived for the Crab cannot be reproduced by any realistic entropy burning outside the iron core, and neutrino-neutronization obtained in electron capture models remains the only viable explanation.
\end{abstract}

Key words: nuclear reactions, nucleosynthesis, abundances - supernovae: general - supernovae: individual (SN 2012ec, Crab, SN 1987A)

\section{INTRODUCTION}

Core-collapse supernovae (CCSNe) are the explosive deaths of massive stars $\left(M_{\text {ZAMS }} \gtrsim 8 M_{\odot}\right)$. As the core of the star collapses to a neutron star or a black hole, a shock wave ejects the mantle and envelope at high velocities. Nucleosynthesis products from hydrostatic and explosive burning are dispersed into the interstellar medium, providing a major production channel for the metals in the universe.

Comparison of spectral models of standard stellar evolution and explosion models with observed nebular CCSN spectra shows encouraging agreement (e.g., Dessart \& Hillier 2011; Jerkstrand et al. 2012, 2014). However, the nature of the explosion mechanism remains unclear. One promising scenario is that of delayed neutrino-driven explosions (see e.g., Nordhaus et al. 2010; Janka et al. 2012; Bruenn et al. 2013; Couch 2013, and references therein). An important test for such models is detailed comparison of explosive nucleosynthesis yields with those inferred from observed nebular-phase spectra. A new method to determine the ratio of the yields of nickel and iron, after radioactive decays, was presented by Jerkstrand et al. (2015, J15 hereafter) and applied to several CCSNe. Together with literature values, a picture emerged in which several CCSNe show $\mathrm{Ni} / \mathrm{Fe}$ ratios that are far above the solar ratio. It is of interest to consider how such $\mathrm{Ni} / \mathrm{Fe}$ ratios arise, and whether they offer constraints on explosion models.

The shock front that travels through the star after the core has collapsed compresses and heats the overlying layers. The innermost layers experience explosive silicon burning, which produces iron-group elements such as ${ }^{56} \mathrm{Ni}$, which powers much of the electromagnetic display of the $\mathrm{SN}$ through its decay to ${ }^{56} \mathrm{Co}$ and ${ }^{56} \mathrm{Fe}$. Silicon burning also produces other isotopes such as ${ }^{57} \mathrm{Ni},{ }^{58} \mathrm{Ni}$, and ${ }^{44} \mathrm{Ti}$, in amounts that depend on the detailed properties of the progenitor structure and the thermodynamic conditions. Diagnosis of the yields of these isotopes can thus provide constraints on the core-collapse process (e.g., Hashimoto et al. 1989; Thielemann et al. 1990, 1996; Woosley \& Hoffman 1991; Grefenstette et al. 2014; Seitenzahl et al. 2014; Perego et al. 2015).

Pioneering calculations of explosive silicon burning were undertaken by Truran et al. (1966, 1967), and the process was expounded upon by Woosley et al. (1973). A key property of the burning in typical core-collapse environments is that it occurs on a sufficiently short timescale $(\lesssim 1 \mathrm{~s})$ that weak reactions have little time to have a significant influence (Fowler $\&$ Hoyle 1964). The proton and neutron numbers are therefore preserved, and as most progenitor layers have about equal numbers of protons and neutrons, the ash is ${ }^{56} \mathrm{Ni}$ rather than ${ }^{56} \mathrm{Fe}$. The neutron content of the fuel can be characterized by the neutron excess $\eta=\left(N_{\mathrm{n}}-N_{\mathrm{p}}\right) /\left(N_{\mathrm{n}}+N_{\mathrm{p}}\right)$, where $N_{\mathrm{n}}$ and $N_{\mathrm{p}}$ are the numbers of neutrons and protons, or equivalently by the electron to baryon fraction $Y_{\mathrm{e}}=(1-\eta) / 2$. For many regimes the burning passes through a phase of quasiequilibrium that has weak sensitivity to the initial composition but a large sensitivity to the value of $\eta$ (Bodansky et al. 1968; 
Woosley \& Hoffman 1992; Hix \& Thielemann 1996, 1999; Meyer et al. 1998; The et al. 1998; Magkotsios et al. 2010, 2011). There is therefore a direct link between observed iron-group yields and three fundamental properties of the explosion: the temperature, the density, and the neutron excess of the fuel. As common 1D progenitor models show the neutron excess to vary significantly with mass coordinate (e.g., Thielemann et al. 1990, 1996; Woosley \& Heger 2007, WH07 henceforth), this offers a potential method for constraining which layers are ejected and which are not. Determination of this "mass cut" in turn reveals information on the nature of the compact remnant and the explosion mechanism.

In this work we explore what constraints can be derived from measured yields of iron and nickel, as are now available for several CCSNe (see J15 and references therein). Under most burning conditions, the iron comes predominantly from ${ }^{56} \mathrm{Ni}$, and the nickel comes predominantly from ${ }^{58} \mathrm{Ni}$. We focus in particular on SN 2012ec, a SN IIP with a progenitor detection (Maund et al. 2013), a well-sampled light curve (Barbarino et al. 2015), and detection of stable nickel lines in the nebular spectrum (J15). In Section 2 we investigate constraints on the nucleosynthesis obtained from parameterized thermodynamic trajectories over an extensive peak temperature-peak density plane. In Section 3 we consider what progenitor layers undergo silicon burning in spherically symmetric stellar evolution and explosion models. In Section 4 we discuss the effects of asymmetries and neutrino processing, as well as implications of the $\mathrm{Ni} / \mathrm{Fe}$ ratios measured in SN 1987A and the Crab, and in Section 5 we summarize our findings.

\section{PARAMETERIZED NUCLEOSYNTHESIS MODELS}

We explore models for nickel and iron production using standard, parameterized thermodynamic trajectories. This single-zone approach assumes that a passing shock wave heats material to a peak temperature $T_{\mathrm{p}}$ and compresses it to a peak density $\rho_{p}$. The material then expands and cools on a prescribed trajectory until the temperature and density are reduced to the extent that nuclear reactions cease (freeze-out). Here, we use adiabatic (constant $T^{3} / \rho$ ) expansion trajectories (Arnett 1971; Woosley et al. 1973)

$$
\begin{aligned}
\frac{d T}{d t}=-\frac{T}{3 \tau} & \frac{d \rho}{d t}=-\frac{\rho}{\tau} \\
T(t)=T_{\mathrm{p}} \exp (-t / 3 \tau) & \rho(t)=\rho_{\mathrm{p}} \exp (-t / \tau)
\end{aligned}
$$

with a static free-fall timescale for the expanding ejecta (Fowler \& Hoyle 1964)

$$
\tau=\left(24 \pi G \rho_{\mathrm{p}}\right)^{-1 / 2} \approx 446 / \rho_{\mathrm{p}}^{1 / 2} \mathrm{~s}
$$

Magkotsios et al. (2010) demonstrate by comparison with trajectories from several core-collapse simulations that nucleosynthesis yields are generally accurate to within a factor 2 using this treatment. One may also use $\rho(t)$ in the expansion timescale instead of the peak density $\rho_{\mathrm{p}}$; see Magkotsios et al.

\section{(2010) for examples of such models.}

Using the code described in Magkotsios et al. (2010, see also Magkotsios et al. 2011), we calculate the mass fractions of nuclear isotopes produced by nuclear burning for different values of $T_{\mathrm{p}}, \rho_{\mathrm{p}}$, and initial electron fraction $Y_{\mathrm{e}}$. We chose peak temperatures and peak densities spanning the range of
$4 \times 10^{9} \leqslant T_{\mathrm{p}} \leqslant 10 \times 10^{9} \mathrm{~K}, \quad 10^{4} \leqslant \rho_{\mathrm{p}} \leqslant 10^{10} \mathrm{~g} \mathrm{~cm}^{-3}$. This parameter space covers the conditions encountered in most CCSN models that produce any significant amount of $\mathrm{Fe}$ or $\mathrm{Ni}$ isotopes. The parameter space is sampled with 121 logaritmically spaced points, so for any value of $Y_{\mathrm{e}}$ we compute the final nucleosynthesis at $121 \times 121$ points in the $\left(T_{\mathrm{p}}, \rho_{\mathrm{p}}\right)$ plane.

Our initial composition is the mixture of ${ }^{28} \mathrm{Si}$ and neutrons that give the specified $Y_{\mathrm{e}}$; this is achieved by mass fractions $X\left({ }^{28} \mathrm{Si}\right)=1-\eta$ and $X(n)=\eta$. The choice of initial composition is not important for vast regions of the chosen thermodynamic parameter space (using e.g., ${ }^{28} \mathrm{Si}$ and ${ }^{29} \mathrm{Si}$ to set $Y_{\mathrm{e}}$ gives the same results) because memory of the initial composition is quickly erased (Magkotsios et al. 2010).

For the $Y_{\mathrm{e}}$ parameter, we focus attention on $Y_{\mathrm{e}}=0.490$, $0.495,0.497$, and 0.499 as these values are representative of different shells in the pre-supernova $(\mathrm{SN})$ progenitor structure (see Section 3). Although there are deep-lying layers in the progenitor with $Y_{\mathrm{e}}$ less than 0.490 , this value marks the lower limit below which ${ }^{56} \mathrm{Ni}$ is no longer the major nucleus produced (e.g., Thielemann et al. 1990), and is therefore of limited interest for this study where we explore $\mathrm{SNe}$ with significant ${ }^{56} \mathrm{Ni}$ production. No candidate fuel for silicon burning has $Y_{\mathrm{e}} \gtrsim 0.499$, and higher values than this are not explored.

\subsection{Overview of Results}

Figure 1 shows the resulting $\mathrm{Ni} / \mathrm{Fe}$ mass ratio (after radioactive decays) in the $\left(T_{\mathrm{p}}, \rho_{\mathrm{p}}\right)$ plane for $Y_{\mathrm{e}}=0.490$, $0.495,0.497$, and 0.499 . Contour lines are drawn at $\mathrm{Ni} /$ $\mathrm{Fe}=0.13$ and 0.27 , bracketing the range derived for SN 2012ec in J15 (compare with the solar ratio of 0.056, Lodders 2003). For Ni/Fe ratios matching SN 2012ec, the nickel yield is dominated by ${ }^{58} \mathrm{Ni}$, and the iron yield is dominated by ${ }^{56} \mathrm{Ni}+{ }^{54} \mathrm{Fe}$, with ${ }^{56} \mathrm{Ni}$ usually the more abundant. We can therefore reach an understanding of the behavior of the $\mathrm{Ni} / \mathrm{Fe}$ ratio by looking at the behavior of these three isotopes.

Figure 2 shows the mass fractions of ${ }^{56} \mathrm{Ni},{ }^{54} \mathrm{Fe},{ }^{58} \mathrm{Ni}$, and ${ }^{4} \mathrm{He}$, for the case $Y_{\mathrm{e}}=0.490$ (other $Y_{\mathrm{e}}$ values give qualitatively similar trends). There are three main production regimes. At $T_{9} \lesssim 4.5\left(T_{9}=T_{\mathrm{p}} / 10^{9} \mathrm{~K}\right)$ is the incomplete burning regime where little iron-group production occurs. At $T_{9} \gtrsim 4.5, \log \rho_{\mathrm{p}} \gtrsim 7$ is the complete burning regime with normal freeze-out. ${ }^{9}$ In this regime burning is well described by a single quasi-static equilibrium (QSE) cluster, in which abundances are largely determined by nuclear Q-values. At $T_{9} \gtrsim 4.5, \log \rho_{\mathrm{p}} \lesssim 7$ is the complete burning regime with $\alpha$ rich freeze-out, where high production of $\alpha$-particles depletes iron-group yields. Burning is now described by two separate QSE clusters, because the triple-alpha reaction has fallen out of equilibrium. The transition region between the normal and $\alpha$ rich freeze-out regimes is called the chasm region. At low densities $\left(\log \rho_{\mathrm{p}} \lesssim 6\right)$ and high temperatures $\left(T_{9} \gtrsim 7\right), Y_{\mathrm{e}}$ increases during the burning due to weak interactions and mostly protons and $\alpha$-particles are produced (the so-called $\alpha p$ regime). For a more refined description of the different burning regimes see Magkotsios et al. (2010).

\footnotetext{
9 We adopt here a helium mass fraction $X\left({ }^{4} \mathrm{He}\right) \approx 0.1$ as the dividing line between "normal" and "alpha-rich" freeze-out regimes.
} 

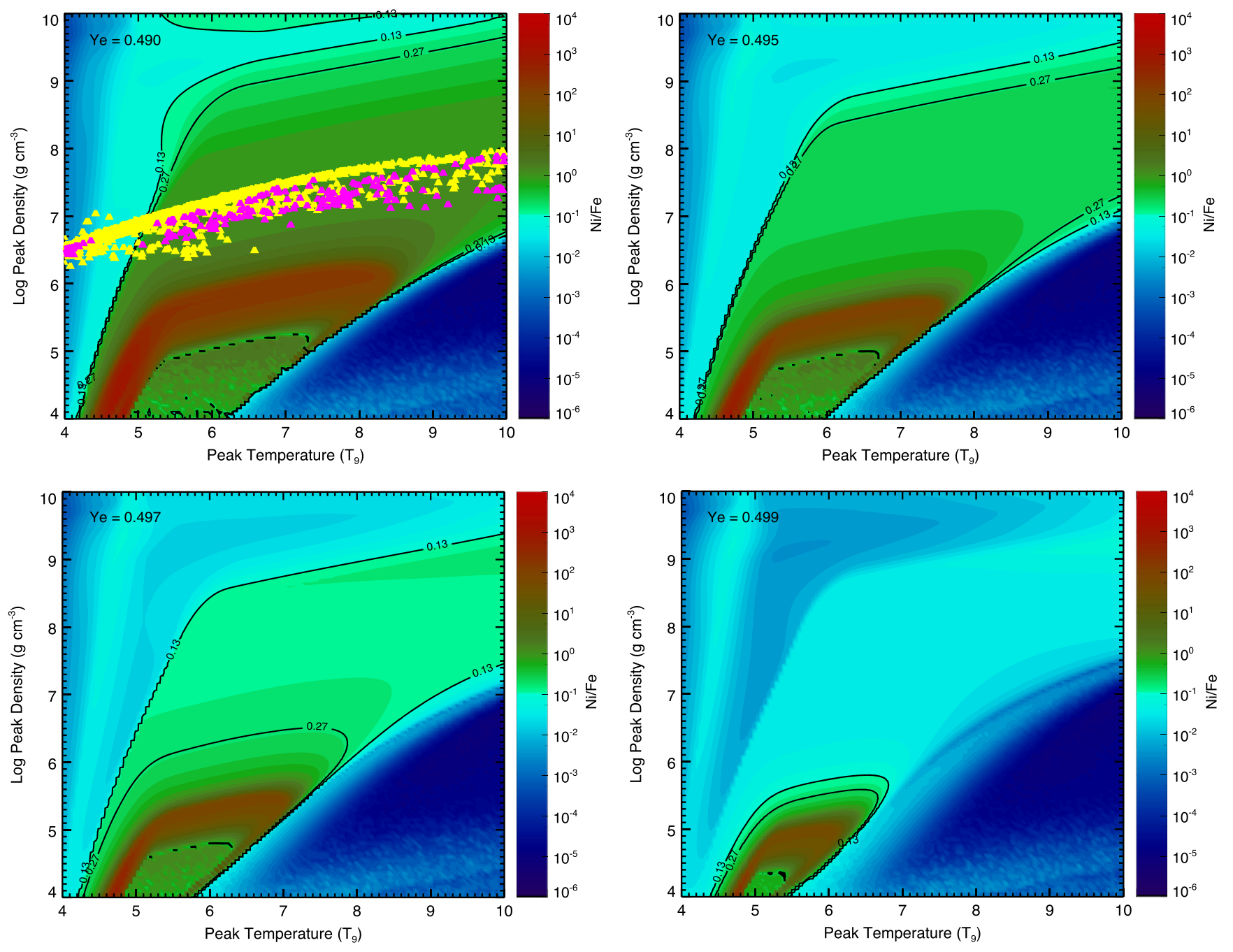

Figure 1. Ni/Fe mass ratio (after radioactive decays) in the $\left(T_{\mathrm{p}}, \rho_{\mathrm{p}}\right)$ plane, for an exponential expansion starting with $Y_{\mathrm{e}}=0.490$ (top left), $Y_{\mathrm{e}}=0.495$ (top right), $Y_{\mathrm{e}}=0.497$ (bottom left), and $Y_{\mathrm{e}}=0.499$ (bottom right). On the $x$-axis $T_{9}=T_{\mathrm{p}} / 10^{9} \mathrm{~K}$. Black contour lines delineate the value range for SN 2012ec (0.13-0.27). Also plotted in the upper left panel are locations of different mass elements in multidimensional explosion simulations of SN 1987A (yellow, Wongwathanarat et al. 2010) and of a $27 M_{\odot}$ star (pink, Müller et al. 2012).

Figure 3 shows the radiation entropy $\log \left(S_{\gamma} / R\right)$, where $S_{\gamma}=4 a T^{3} /(3 \rho)$ and $R$ is the gas constant. The entropy values will be of use for later discussion.

Figure 2 allows a quantitative description of the ${ }^{56} \mathrm{Ni},{ }^{54} \mathrm{Fe}$, and ${ }^{58} \mathrm{Ni}$ yields, and from them the ratio $\mathrm{Ni} / \mathrm{Fe} \approx{ }^{58} \mathrm{Ni} /\left({ }^{54} \mathrm{Fe}+\right.$ ${ }^{56} \mathrm{Ni}$. The neutron excess of ${ }^{56} \mathrm{Ni}$ is zero, whereas for ${ }^{54} \mathrm{Fe}$ and ${ }^{58} \mathrm{Ni}$ it is 0.037 and 0.034 , respectively. Thus, as long as these three isotopes dominate the composition, the neutron excess of the matter must be stored in some combination of ${ }^{54} \mathrm{Fe}$ and ${ }^{58} \mathrm{Ni}$. Their mass fractions $X$ will then be constrained by

$$
\eta=0.037 X\left({ }^{54} \mathrm{Fe}\right)+0.034 X\left({ }^{58} \mathrm{Ni}\right) .
$$

The maximum mass fractions of these two isotopes are thus $X\left({ }^{54} \mathrm{Fe}\right)<\eta / 0.037$ and $X\left({ }^{58} \mathrm{Ni}\right)<\eta / 0.034$. The peak plateau values of ${ }^{54} \mathrm{Fe}$ and ${ }^{58} \mathrm{Ni}$ production in Figure 2 correspond to the mass fractions that give the correct neutron excess when that isotope dominates the composition.

The neutron excess is predominantly stored in ${ }^{54} \mathrm{Fe}$ at low entropy and in ${ }^{58} \mathrm{Ni}$ at high entropy. In the normal freeze-out regime, the $\mathrm{Ni} / \mathrm{Fe}$ ratio must therefore increase with increasing entropy as ${ }^{54} \mathrm{Fe}$ is replaced by ${ }^{58} \mathrm{Ni}$. When the entropy is large enough, both ${ }^{56} \mathrm{Ni}$ and ${ }^{58} \mathrm{Ni}$ are replaced by $\alpha$-particles. For high temperatures, weak interactions de-neutronize the matter and a decrease in the $\mathrm{Ni} / \mathrm{Fe}$ yield follows in the $\alpha p$ regime. At lower temperatures, where the original neutron excess is maintained, the $\mathrm{Ni} / \mathrm{Fe}$ ratio stays high as ${ }^{56} \mathrm{Ni}$ is destroyed more efficiently than ${ }^{58} \mathrm{Ni}$ in strong $\alpha$-rich freeze-out.

$$
\text { 2.2. } Y_{\mathrm{e}}=0.490
$$

The $\mathrm{Ni} / \mathrm{Fe}$ ratio at $Y_{\mathrm{e}}=0.490$ is shown in Figure 1 (top left). In the complete burning regime, there is a minimum for the ratio at entropy $\log \left(S_{\gamma} / R\right) \approx-2$; at lower entropy the ${ }^{58} \mathrm{Ni}$ yield increases due to electron captures which lower $Y_{\mathrm{e}}$, whereas at higher entropies it increases as ${ }^{58} \mathrm{Ni}$ replaces ${ }^{54} \mathrm{Fe}$ as the main storage nucleus for neutron excess. This growth continues well into the $\alpha$-rich freeze-out regime $\left(\log \left(S_{\gamma} / R\right) \gtrsim+0.5\right)$, but is quenched in the $\alpha p$ regime. The maximum ${ }^{56} \mathrm{Ni}$ production is only $\sim 40 \%$ as ${ }^{54} \mathrm{Fe}$ and ${ }^{58} \mathrm{Ni}$ are produced in large amounts at these relatively large neutron-rich compositions. 

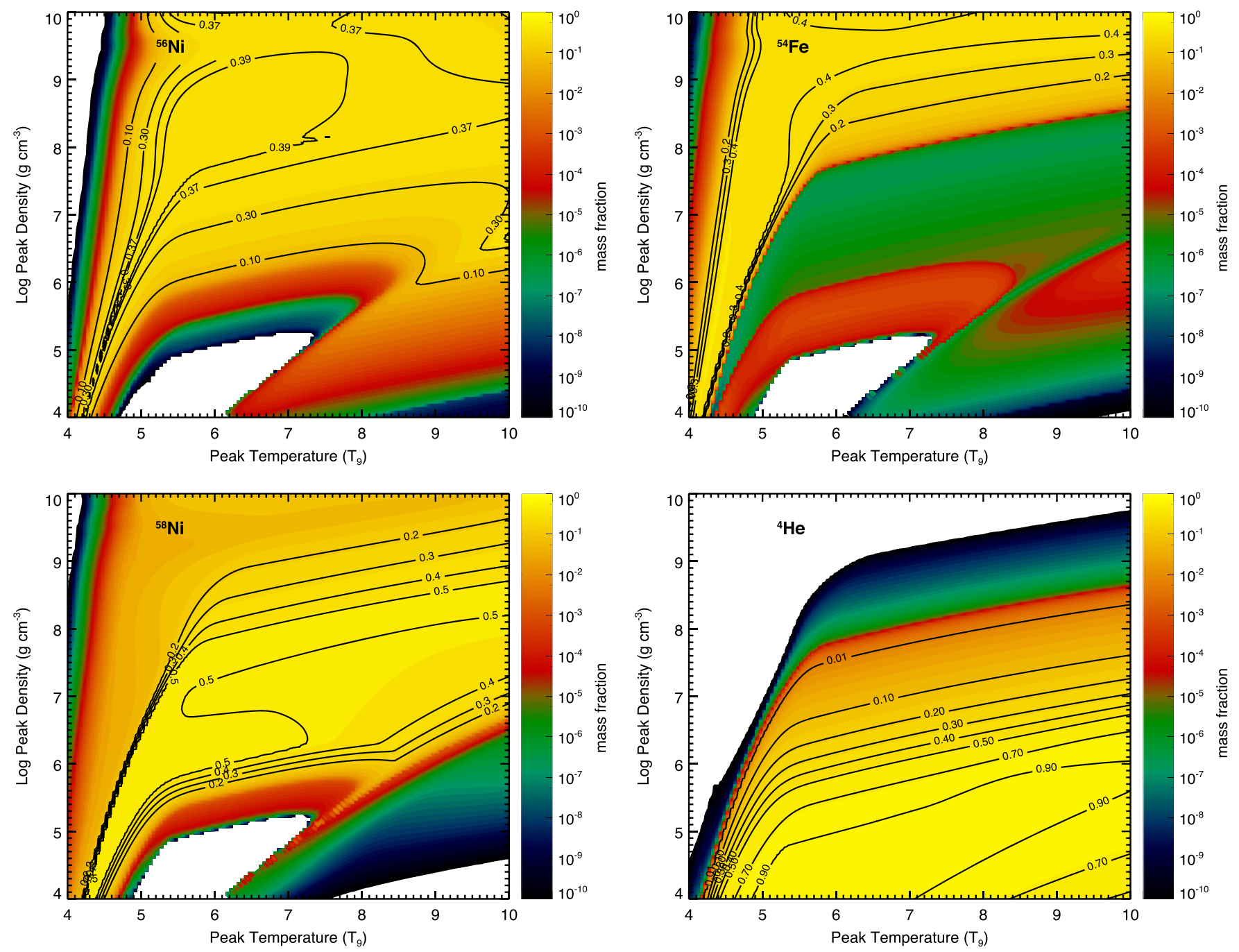

Figure 2. Mass fractions of ${ }^{56} \mathrm{Ni}$ (top left), ${ }^{54} \mathrm{Fe}$ (top right), ${ }^{58} \mathrm{Ni}$ (bottom left), and ${ }^{4} \mathrm{He}$ (bottom right), for an initial $Y_{\mathrm{e}}=0.490$.

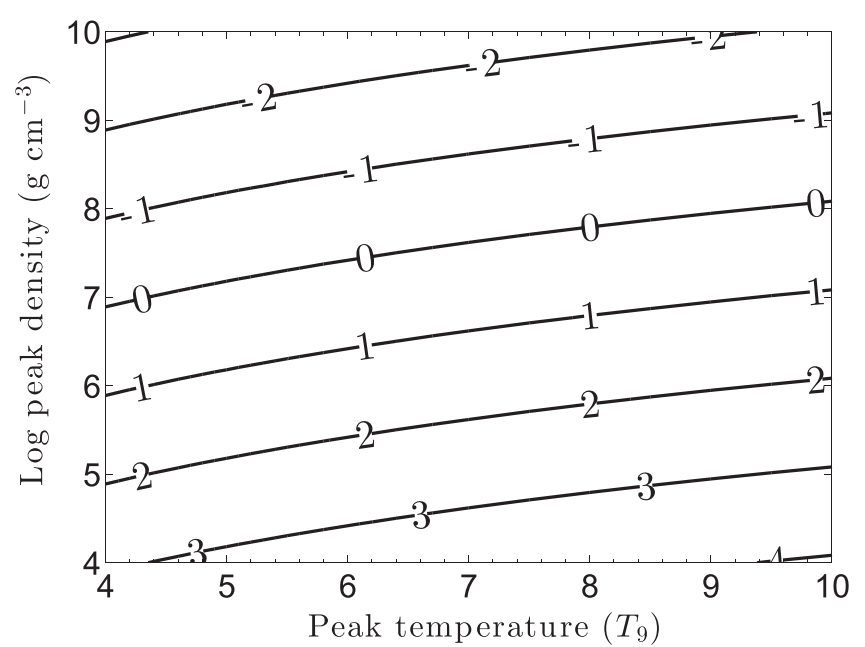

Figure 3. Radiation entropy, $\log S_{\gamma} / R$.

The band bounded by the contour lines for SN 2012ec lies in the normal freeze-out regime. Entrance and exit into the allowed band (along with increasing entropy) are driven by switchover between ${ }^{54} \mathrm{Fe}$ and ${ }^{58} \mathrm{Ni}$, while ${ }^{56} \mathrm{Ni}$ changes little.
Using Equation (4) with $\eta=0.02$ gives $X\left({ }^{54} \mathrm{Fe}\right)<0.54$ and $X\left({ }^{58} \mathrm{Ni}\right)<0.59$. The large plateau region of maximum ${ }^{58} \mathrm{Ni}$ production thus gives a $\mathrm{Ni} / \mathrm{Fe}$ ratio $\gtrsim 1\left(X\left({ }^{56} \mathrm{Ni}\right) \lesssim 0.4\right)$, over 18 times the solar value.

In the incomplete burning regime $\left(T_{9} \lesssim 5\right)$, the allowed strip between the $\mathrm{Ni} / \mathrm{Fe}=0.13$ and 0.27 contours is very narrow and it is unlikely that the burning in SN 2012ec occurred precisely under these conditions. The same conclusion can be drawn for the narrow strip delineating the transition into the $\alpha p$ regime.

$$
\text { 2.3. } Y_{\mathrm{e}}=0.495
$$

At $Y_{\mathrm{e}} \geqslant 0.495(\eta \leqslant 0.01)$, the neutron excess is too small for ${ }^{54} \mathrm{Fe}(\eta=0.037)$ and ${ }^{58} \mathrm{Ni}(\eta=0.034)$ to dominate the composition, and ${ }^{56} \mathrm{Ni}(\eta=0)$ is the most abundant irongroup nucleus produced. The $Y_{\mathrm{e}}=0.495$ calculation is shown in Figure 1, top right. The $\mathrm{Ni} / \mathrm{Fe}$ band allowed by the SN 2012ec abundance determinations moves to slightly higher entropies relative to the $Y_{\mathrm{e}}=0.490$ case. Note how the Ni/ $\mathrm{Fe}=0.27$ contour is beginning to move away from the $\alpha \mathrm{p}$ regime. The width of this lower density band is not significant at $Y_{\mathrm{e}}=0.495$, but will continue to widen and become significant as $Y_{\mathrm{e}}$ increases. The plateau region of maximum 
${ }^{58} \mathrm{Ni}$ production has $X\left({ }^{58} \mathrm{Ni}\right)=0.29$, giving about six times solar $\mathrm{Ni} / \mathrm{Fe}$.

$$
\text { 2.4. } Y_{\mathrm{e}}=0.497
$$

The $Y_{\mathrm{e}}=0.497$ calculation is shown in Figure 1, bottom left. This $Y_{\mathrm{e}}$ is interesting because it provides a very large region in thermodynamic space where the inferred $\mathrm{Ni} / \mathrm{Fe}$ ratio of $\mathrm{SN} 2012 \mathrm{ec}$ is produced. The allowed $\mathrm{Ni} / \mathrm{Fe}$ band covers entropies $-1<\log \left(S_{\gamma} / R\right)<+1$, and crosses into the $\alpha$-rich freeze-out regime. A transition into the allowed band from low to high entropy occurs again as the ${ }^{58} \mathrm{Ni}$ abundance increases when the entropy increases past $\log \left(S_{\gamma} / R\right) \sim-1$ (normal freeze-out). However, the ${ }^{58} \mathrm{Ni}$ abundance now levels off to its maximum value $X\left({ }^{58} \mathrm{Ni}\right)=0.18$ (from Equation (4)) before the upper boundary $(\mathrm{Ni} / \mathrm{Fe}=0.27)$ of the regime is crossed, and gives a $\mathrm{Ni} / \mathrm{Fe}$ ratio that lies within the tolerance interval for SN 2012ec, explaining the large size of the allowed region at this $Y_{\mathrm{e}}$.

A qualitative difference to lower $Y_{\mathrm{e}}$ values is that now a part of the allowed band lies in the $\alpha$-rich freeze-out regime. In particular, the upper $\mathrm{Ni} / \mathrm{Fe}$ boundary $(0.27)$ is crossed (with increasing entropy) not as ${ }^{58} \mathrm{Ni}$ increases by too much (as at lower $Y_{\mathrm{e}}$ ), but by a more rapid depletion of ${ }^{56} \mathrm{Ni}$ compared to ${ }^{58} \mathrm{Ni}$ in the $\alpha$-rich freeze-out regime.

A ${ }^{58} \mathrm{Ni} /{ }^{56} \mathrm{Ni}$ ratio of 0.2 corresponds to an electron fraction $Y_{\mathrm{e}}=0.497$ if the abundances of other nuclei are negligible. If the freeze-out composition is dominated by these two isotopes, then $Y_{\mathrm{e}}=0.497$ must be the electron fraction of the fuel (assuming $Y_{\mathrm{e}}$ stays constant during the burning). If other isotopes are present at freeze-out, but ${ }^{56} \mathrm{Ni}$ still dominates the mass fraction, $Y_{\mathrm{e}}=0.497$ represents the maximum allowed electron fraction, as no significant amounts of proton-rich isotopes $\left(Y_{\mathrm{e}}>0.5\right)$ are produced and the other isotopes must contribute zero or positive neutron excess. However, incomplete burning or a strong $\alpha$-rich freeze-out may allow also larger $Y_{\mathrm{e}}$ values as ${ }^{56} \mathrm{Ni}$ is then not the main nucleus produced.

$$
\text { 2.5. } Y_{\mathrm{e}}=0.499
$$

The $Y_{\mathrm{e}}=0.499$ calculation is shown in Figure 1, bottom right. There is no regime at normal freeze-out that produces enough ${ }^{58} \mathrm{Ni}$ to reproduce the $\mathrm{SN} 2012 \mathrm{ec} \mathrm{Ni} / \mathrm{Fe}$ ratio, as the maximum ${ }^{58} \mathrm{Ni}$ fraction $X\left({ }^{58} \mathrm{Ni}\right)=0.06$ (from Equation (4)), and thus $\mathrm{Ni} / \mathrm{Fe} \lesssim 0.06$ (as long as ${ }^{56} \mathrm{Ni}$ dominates). The only allowed band is restricted to a narrow region in the $\alpha$-rich freeze-out regime with densities $\log \rho_{\mathrm{p}}<5.5$. The allowed band has an entropy of $\log \left(S_{\gamma} / R\right)=1.5-1.7$. At these peak initial conditions $\alpha$-particles dominate the final composition, and $X\left({ }^{56} \mathrm{Ni}\right) \lesssim 0.3$. The allowed band is reached as ${ }^{56} \mathrm{Ni}$ is depleted more strongly than ${ }^{58} \mathrm{Ni}$ in the $\alpha$-rich freeze-out.

\subsection{Relation to Progenitor Density and Shock Velocity}

Our $Y_{\mathrm{e}}$ sensitivity study suggests two fundamentally different ways that a $\mathrm{Ni} / \mathrm{Fe}$ ratio of $\sim 0.2$ can be achieved. The first is a normal freeze-out burning at low entropies $\left(-1 \lesssim \log \left(S_{\gamma} / R\right) \lesssim 0\right)$ of a high neutron excess fuel $\left(Y_{\mathrm{e}}=0.490-0.497\right)$. The second is an $\alpha$-rich freeze-out burning at high entropies $\left(\log \left(S_{\gamma} / R\right) \gtrsim 0\right)$ of a lower neutron excess fuel $\left(Y_{\mathrm{e}}=0.497-0.499\right)$. At normal freeze-out, the $\mathrm{Ni} /$ $\mathrm{Fe}$ ratio grows with entropy because the ${ }^{58} \mathrm{Ni}$ abundance grows
Table 1

Constraints on $\rho_{\text {pre }}$ and $v_{\mathrm{s}}$ in SN 2012ec from Application of the Strong Shock Conditions

\begin{tabular}{lcc}
\hline \hline$Y_{\mathrm{e}}$ & $\log \rho_{\text {pre }}\left(\mathrm{g} \mathrm{cm}^{-3}\right)$ & $\log v_{\mathrm{s}}\left(\mathrm{km} \mathrm{s}^{-1}\right)$ \\
\hline 0.490 & $7.2-9.2$ & $3.2-3.6$ \\
0.495 & $7.1-8.7$ & $3.4-3.6$ \\
0.497 & $5.2-8.2$ & $3.6-4.6$ \\
0.499 & $3.2-5.0$ & $5.0-5.5$ \\
\hline
\end{tabular}

(at the expense of ${ }^{54} \mathrm{Fe}$ ), whereas in an $\alpha$-rich freeze-out the $\mathrm{Ni} /$ Fe ratio grows because ${ }^{56} \mathrm{Ni}$ is depleted more efficiently than ${ }^{58} \mathrm{Ni}$ with increasing entropy.

In the non-relativistic, strong shock limit for a radiationdominated gas (adiabatic index $\gamma=4 / 3$ ), the post-shock conditions are related to pre-shock conditions by (e.g., Sedov 1959; Chevalier 1976)

$$
\begin{aligned}
\rho_{\text {post }} & =\left(\frac{\gamma+1}{\gamma-1}\right) \rho_{\text {pre }}=7 \rho_{\text {pre }} \\
T_{\text {post }} & =4300 \rho_{\text {pre }}^{1 / 4} v_{\mathrm{s}}^{1 / 2} \mathrm{~K},
\end{aligned}
$$

where $v_{\mathrm{s}}$ is the shock speed. Thus, a $\left[T_{\text {post }}, \rho_{\text {post }}\right]$ pair maps onto a $\left[\rho_{\text {pre }}, v_{\mathrm{s}}\right]$ pair as

$$
\begin{aligned}
\rho_{\text {pre }} & =\frac{1}{7} \rho_{\text {post }} \\
v_{\mathrm{s}} & =\left(\frac{T_{\text {post }}}{4300 \mathrm{~K}}\right)^{2}\left(\frac{1}{7} \rho_{\text {post }}\right)^{-1 / 2} \mathrm{~cm} \mathrm{~s}^{-1} .
\end{aligned}
$$

By equating the post-shock conditions with the peak conditions in the nucleosynthesis models (i.e., $T_{\text {post }}=T_{\mathrm{p}}$ and $\rho_{\text {post }}=\rho_{\mathrm{p}}$ ), a determined $\mathrm{Ni} / \mathrm{Fe}$ ratio constrains the allowed ranges of $\rho_{\text {pre }}$ and $v_{\mathrm{s}}$; the values for $\mathrm{SN} 2012 \mathrm{ec}$ are listed in Table 1. More neutron-rich (lower $Y_{\mathrm{e}}$ ) material requires higher pre-SN densities and lower shock speeds to achieve the same $\mathrm{Ni} / \mathrm{Fe}$ production. Note that the $Y_{\mathrm{e}}=0.499$ case requires shock speeds exceeding $c / 3$. Electron capture $\mathrm{SNe}$ are capable of achieving shock speeds around this mark as the shock accelerates down their extremely steep density gradients (Janka et al. 2008). But SN 2012ec, or any other CCSN producing $M\left({ }^{56} \mathrm{Ni}\right) \gg 0.01 M_{\odot}$, must arise from a more massive progenitor, and the cores of these are shallower (approximately $\left.\rho \propto r^{-3}\right)$, which allows no significant shock acceleration (Matzner \& McKee 1999). The initial velocity scale of the shock is limited to $v_{\mathrm{s}} \sim \sqrt{2 E / M} \lesssim 0.1 c$ from the release of 8.8 MeV per baryon in fusion reactions (see Scheck et al. 2006 for a discussion of the energy budget of the explosion). In a self-regulating explosion mechanism, such as the neutrinodriven one, the shock can also never significantly exceed the escape velocity as the energy deposition shuts off as soon as that happens. This gives a constraint $v_{\mathrm{s}} \lesssim \sqrt{2 G M / R} \lesssim 0.2 c$ for $M=1.4 M_{\odot}$ and $R=150 \mathrm{~km}$ (typical shock radius prior to explosion). We conclude that the $Y_{\mathrm{e}}=0.499$ case has no realistic shock scenario associated with it, and can be ruled out as a viable scenario for silicon burning in SN 2012ec.

The $Y_{\mathrm{e}} \leqslant 0.497$ scenarios all require physically reasonable progenitor densities and shock speeds. We consider, however, that $Y_{\mathrm{e}} \leqslant 0.495$ is unlikely because the required entropies are over an order of magnitude lower than encountered in typical 


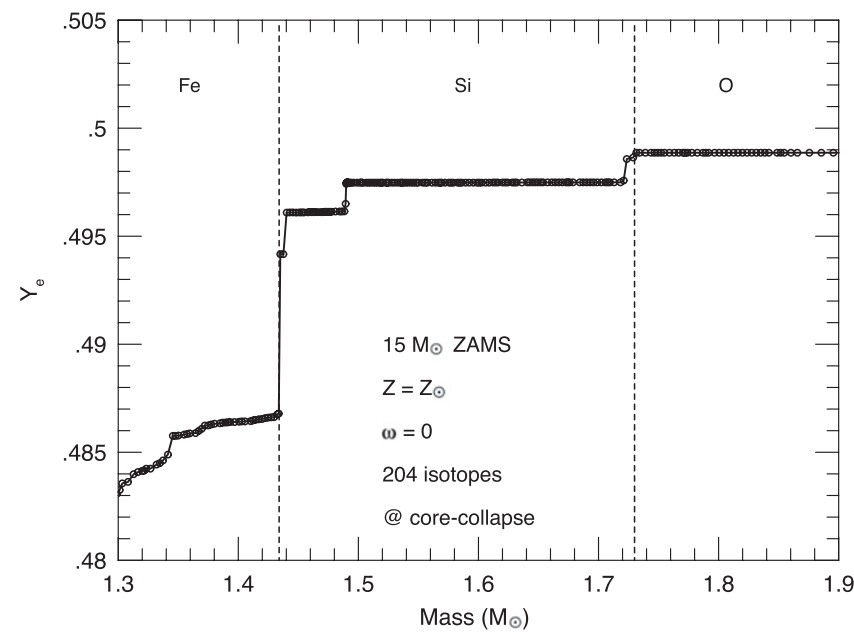

Figure 4. $Y_{\mathrm{e}}$ profile at core collapse of a solar metallicity $15 M_{\odot}$ MESA model. The $\mathrm{Fe}, \mathrm{Si}$, and $\mathrm{O}$ composition layers are labelled, and open circles mark the locations of grid points in the model.

core-collapse explosion simulations (a few examples of temperatures and densities obtained in core-collapse simulations are plotted in the upper left panel of Figure 1). The much larger regime in thermodynamic space at $Y_{\mathrm{e}}=0.497$ instead overlaps with typical explosion entropies. We therefore conclude that the most natural scenario in which the $\mathrm{Ni} / \mathrm{Fe}$ ratio of SN 2012ec is explained is that the SN burnt and ejected a progenitor layer with $Y_{\mathrm{e}} \approx 0.497$.

\section{STELLAR EVOLUTION AND EXPLOSION MODELS}

So far our analysis has been focused on single-zone models with parameterized thermodynamic trajectories, allowing us to explore how the $\mathrm{Ni} / \mathrm{Fe}$ production ratio depends on burning conditions. In this section, we examine stellar evolution and explosion models from the literature to explore which progenitors and explosions can give the required thermodynamic conditions and neutron excess of the fuel derived in Section 2. This is a challenging step since our understanding of both progenitor evolution and explosion mechanisms is far from complete, but illustrates the role of the $\mathrm{Ni} / \mathrm{Fe}$ ratio as an important constraint for the testing of both current and future $\mathrm{SN}$ models. We limit ourselves in this section to spherically symmetric models, providing some discussion of multidimensional effects in Section 4. We begin by describing the evolution of the neutron excess during the pre-SN evolution.

\subsection{Neutron Excess During pre-SN Evolution}

Figure 4 shows the final $Y_{\mathrm{e}}$ profile prior to core-collapse of a solar metallicity, 201 isotope, $M_{\text {ZAMS }}=15 M_{\odot}$ model calculated with MESA, public release version 7315 (Paxton et al. 2011, 2013). The boundaries of the O, Si, and Fe shells are labeled. Core helium burning increases $\eta$ from its initial value close to zero $\left(Y_{\mathrm{e}}=0.5\right)$ through the sequence ${ }^{14} \mathrm{~N}(\alpha, \gamma){ }^{18} \mathrm{~F}(\beta+){ }^{18} \mathrm{O}$ (e.g., Couch \& Arnett 1972; Arnett \& Thielemann 1985; Thielemann \& Arnett 1985). The ${ }^{18} \mathrm{O}$ is burnt further, but in an $\eta$-preserving way. The final neutron excess of the helium burning ashes is therefore the neutron excess of ${ }^{18} \mathrm{O}(\eta=0.11)$ times the mass fraction of material burnt to ${ }^{18} \mathrm{O}$ (and further), which equals $18 / 14$ times the mass fraction of CNO, as the CNO cycle converts most CNO to ${ }^{14} \mathrm{~N}$, and the burning of ${ }^{14} \mathrm{~N}$ to ${ }^{18} \mathrm{O}$ occurs with close to $100 \%$ efficiency in the core. The CNO mass fraction is about $2 / 3$ of the total metal mass fraction at solar metallicity (Asplund et al. 2009). Assuming this to hold also for other metallicities, we obtain

$$
\eta_{\text {He-burn ash }} \approx 10^{-3} \times\left(\frac{Z}{0.014}\right) .
$$

Core carbon burning further increases $\eta$ through ${ }^{12} \mathrm{C}(p, \gamma){ }^{13} \mathrm{~N}\left(\beta^{+}\right){ }^{13} \mathrm{C}(\alpha, \mathrm{n}){ }^{16} \mathrm{O}$ as well as ${ }^{20} \mathrm{Ne}(n, \gamma){ }^{21} \mathrm{Ne}$ $(p, \gamma)^{22} \mathrm{Na}\left(\beta^{+}\right)^{22} \mathrm{Ne}(\alpha, n)^{25} \mathrm{Mg}(p, \gamma)^{26} \mathrm{Al}\left(\beta^{+}\right)^{26} \mathrm{Mg} \quad$ (Thielemann \& Arnett 1985). The increase in $\eta$ is larger for lowermass cores: about a factor of two for a $4 M_{\odot}$ He core but less for more massive ones. Note that carbon burning will produce a non-zero neutron excess even if $Z=0$. At zero metallicity, Woosley et al. (2002) find $\eta=1.2 \times 10^{-3}$ and $6.8 \times 10^{-4}$ for $15 M_{\odot}$ and $25 M_{\odot}$ progenitors, respectively. As an approximate formula we may take

$$
\eta_{\text {C-burn ash }} \approx 10^{-3}\left(1+\frac{Z}{0.014}\right)
$$

The neutron excess shows no significant changes during core or shell neon burning. Core oxygen burning increases the neutron excess to $\eta=0.01-0.03\left(Y_{\mathrm{e}}=0.485-0.495\right)$ due to electron capture reactions on ${ }^{33} \mathrm{~S},{ }^{35} \mathrm{Cl},{ }^{31} \mathrm{P}$, and ${ }^{32} \mathrm{~S}$, with higher values for lower-mass cores (Thielemann \& Arnett 1985). However, layers later exposed to explosive silicon burning will, for most progenitors and mass cuts, have experienced shell oxygen burning rather than core oxygen burning. The lower density and higher temperature in shell oxygen burning make electron captures less efficient and $\eta$ is smaller (Thielemann \& Arnett 1985; Thielemann et al. 1990; Woosley et al. 2002) (see also Figure 4). Thielemann et al. (1990) obtain $\eta \approx 0.01\left(Y_{\mathrm{e}} \approx 0.495\right)$ in this layer, whereas WH07 have $\eta \approx 4 \times 10^{-3} \quad\left(Y_{\mathrm{e}} \approx 0.498\right)$. In our MESA simulation we obtain $\eta=6 \times 10^{-3}\left(Y_{\mathrm{e}}=0.497\right)$ in this layer. During core silicon burning a large number of electron capture reactions increase $\eta$ to values $\eta \gtrsim 0.06 \quad\left(Y_{\mathrm{e}} \lesssim 0.47\right.$, see Figure 4). Similarly to oxygen burning, shell silicon burning also gives lower neutron excess values, with Thielemann \& Arnett (1985) obtaining $\eta=0.04\left(Y_{\mathrm{e}}=0.48\right)$ in an $8 M_{\odot} \mathrm{He}$ core, similar to the value obtained in the outer Fe core in our MESA calculation for a $15 M_{\odot}$ star $\left(Y_{\mathrm{e}}=0.486\right.$, Figure 4$)$.

Three distinct zones with very different $\eta$ are thus present in the progenitor: the iron core $(\eta \gtrsim 0.04)$, the silicon shell $(\eta \sim 0.01)$, and the oxygen shell $\left(\eta \sim 10^{-3} \times(1+Z / 0.014)\right)$. The inferred $\mathrm{Ni} / \mathrm{Fe}$ ratio in $\mathrm{SN} 2012 \mathrm{ec}$ is produced for $\eta \approx 6 \times 10^{-3}$ (Section 2). There are then two candidate origin sites: (1) the silicon shell; (2) the oxygen shell in a very highmetallicity progenitor, $Z \gtrsim 0.07$. The second scenario requires five times the solar metallicity, much higher than the measured metallicity of $Z=0.014-0.025\left(1-1.8\right.$ times $\left.Z_{\odot}\right)$ in the SN 2012ec region (Ramya et al. 2007). We conclude that $\mathrm{SN} 2012 \mathrm{ec}$, and likely most other $\mathrm{SNe}^{10}$ that produce a Ni/Fe ratio of several times solar, burnt and ejected part of the silicon layer.

\footnotetext{
${ }^{10}$ Even the highest abundances in the inner few kiloparsecs of spiral galaxies such as M101 are at most twice solar (Bresolin 2007) and a value of five times solar would be unprecedented.
} 


\subsection{Explosion}

Explosions in spherical symmetry can be modeled using a thermal bomb (e.g., Thielemann et al. 1996), a piston (e.g., Woosley \& Weaver 1995), or a kinetic bomb (e.g., Chieffi \& Limongi 2013). Young \& Fryer (2007) discuss the differences in the yields between thermal bomb and piston-driven methods. In the specific case of a neutrino-driven mechanism, the explosions can be modeled with self-consistently calculated neutrino luminosities (e.g., Kitaura et al. 2006) or with tunable ones (e.g., Perego et al. 2015).

The explosion mechanism will take some time $t_{\text {delay }}$ after initiation of collapse to send the shock wave off. If we specifically define this time as the time at which the shock wave has expanded enough to give a post-shock temperature below the silicon-burning limit, and denote the radius of the shock at that point as $R_{\mathrm{Si} \text {-burn }}$, the total mass that will have been burnt is

$$
M_{\text {Si-burn }}=\int_{0}^{R_{\mathrm{Si}-\text { burn }}} \rho\left(r, t_{\text {delay }}\right) 4 \pi r^{2} d r .
$$

A quite accurate estimate of the size $R_{\mathrm{Si} \text {-burn }}$ of the region that experiences complete silicon burning can be obtained from the equation (Woosley 1988)

$$
E=\frac{4 \pi}{3} R_{\text {Si-burn }}^{3} a T_{\text {Si-burn }}^{4},
$$

Solving for $R_{\mathrm{Si} \text {-burn }}$ with $T_{\mathrm{Si} \text {-burn }}=5 \times 10^{9} \mathrm{~K}$ gives

$$
R_{\text {Si-burn }}=3700 \mathrm{~km}\left(\frac{E}{10^{51} \mathrm{erg}}\right)^{1 / 3} .
$$

For prompt explosions the relevant density profile is that of the progenitor before any infall, $\rho\left(r, t_{\text {delay }}=0\right)$. If the explosion is delayed, then matter has time to accrete and the amount of mass inside $R_{\text {Si-burn }}$ will be higher. For example, for $M_{\text {ZAMS }}=13$, 15, 20, and $25 M_{\odot}$ progenitors, Thielemann et al. (1996, henceforth T96) find $M_{\mathrm{Si} \text {-burn }}=1.42,1.46,1.70$, and $1.79 M_{\odot}$ for a thermal bomb explosion of $E=1 \mathrm{~B}$ $\left(1 \mathrm{~B}=1 \mathrm{Bethe}=10^{51} \mathrm{erg}\right)$ and a delay time of zero. For a delay time of one second, these masses increase to $M_{\text {Si-burn }}=1.50,1.53,1.77$, and $1.91 M_{\odot}$.

The mass cut $M_{\text {cut }}$ (the dividing point between matter that falls onto the compact remnant and matter that is ejected) cannot be computed ab initio from thermal bomb or pistondriven models. Nevertheless, the ejection of even small amounts of iron core material results in significantly non-solar abundance patterns (Arnett 1996), and one can therefore argue that most $\mathrm{SNe}$ should have their mass cuts above the iron core (which equals the mass coordinate of the inner edge of the silicon shell). This mass coordinate is $M_{\mathrm{Fe}-\text { core }}=1.18,1.28$, $1.40,1.61 M_{\odot}$ for $M_{\mathrm{ZAMS}}=13,15,20,25 M_{\odot}$ in the T96 models.

For a given explosion energy $E$ and density profile at the time of shock passage $\rho\left(r, t_{\text {delay }}\right)$ we can thus approximate $M_{\text {Si-burn }}$ using Equations (9) and (11). For a given ejected ${ }^{56} \mathrm{Ni}$ mass $M_{56 \mathrm{Ni} \text {, jected }}$ the mass cut $M_{\text {cut }}$ is then

$$
M_{\text {cut }}=M_{\text {Si-burn }}-M_{56 \mathrm{Ni}, \text { jected }} \text {, }
$$

where we have assumed that the mass of the silicon-burning ashes is dominated by ${ }^{56} \mathrm{Ni}$. Table 2 lists the resulting mass cuts for different progenitors from the T96 model grid (using
Table 2

Mass Cuts From the T96 Models and $Y_{\mathrm{e}}$

\begin{tabular}{lccc}
\hline \hline$M_{\text {ZAMS }}$ & $M_{\text {Si,out }}$ & $M_{\text {cut }}$ & $Y_{\mathrm{e}}$ \\
\hline 13 & 1.49 & 1.39 & 0.491 \\
15 & 1.37 & 1.43 & 0.499 \\
20 & 1.63 & 1.67 & 0.499 \\
25 & 1.64 & 1.79 & 0.499
\end{tabular}

Note. For four different masses, columns 2, 3, and 4 show the outer mass coordinate for the silicon shell $M_{\mathrm{Si} \text { out }}$ (taken as the point where $Y_{e}$ crosses 0.4985 ), the inferred mass cuts $M_{\text {cut }}$ (assuming zero delay time, $E=1 \mathrm{~B}$, and a Si-burning ash mass of $0.03 M_{\odot}$ ), and the $Y_{e}$ values in the silicon-burning region for those mass cuts.
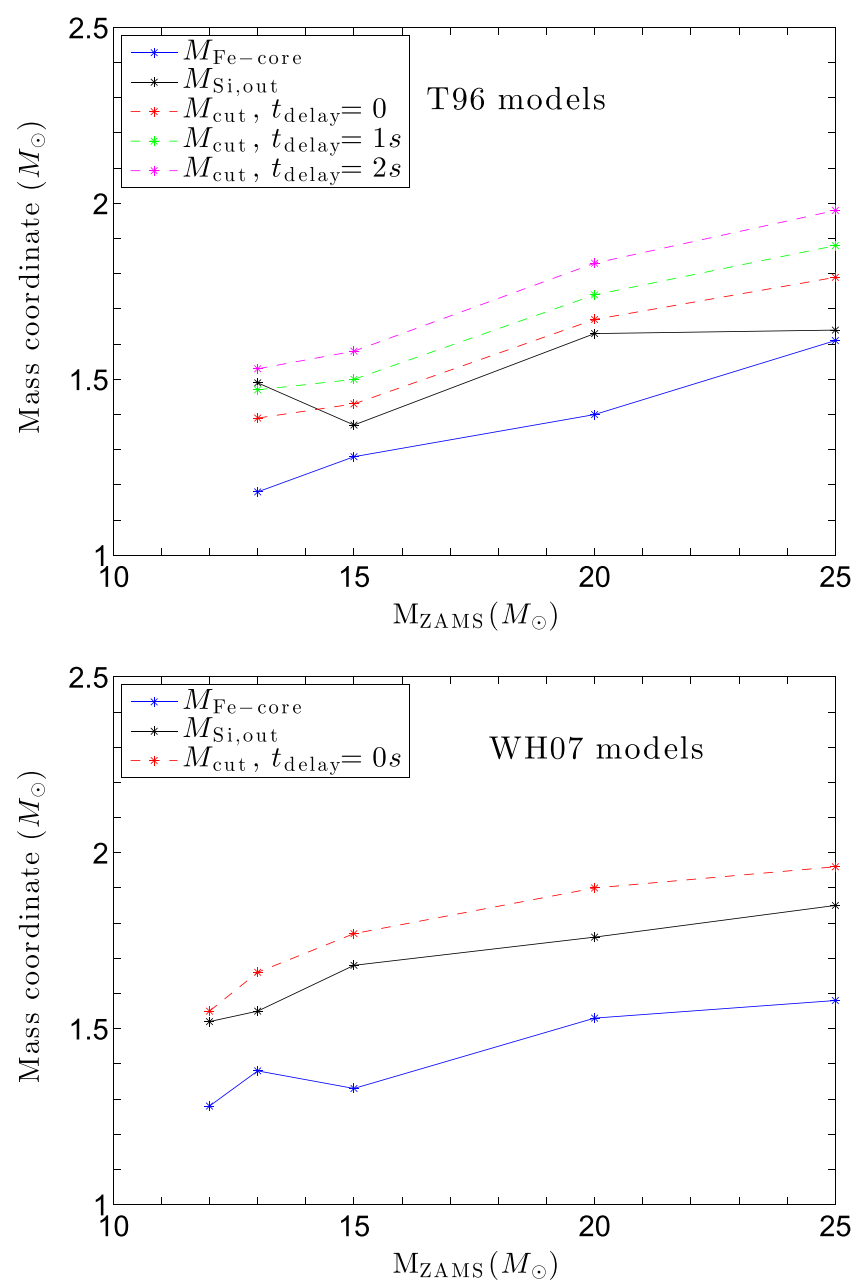

Figure 5. Mass cuts for $E=1 \mathrm{~B}$ and $M\left({ }^{56} \mathrm{Ni}\right)=0.03 M_{\odot}$, compared to the outer edge of the silicon shell $M_{\mathrm{Si} \text {,out }}$ (black, solid), from the T96 models (top) and the WH07 models (bottom). In the T96 models, ejection of silicon-shell fuel occurs only for the $M_{\text {ZAMS }}=13 M_{\odot}$ progenitor. In the WH07 models, silicon-shell material is not ejected for any progenitor mass.

$M_{56 \mathrm{Ni} \text {, ejected }}=0.03 M_{\odot}$ as determined for SN 2012ec), and the $Y_{\mathrm{e}}$ value in the region between $M_{\text {cut }}$ and $M_{\mathrm{Si} \text {-burn }}$ (which becomes the ejected material that has experienced silicon burning).

Figure 5 (top) shows the relevant quantities from the T96 grid, also plotting $M_{\text {cut }}$ for non-zero delay times. For $E \geqslant 1 \mathrm{~B}$, only the combination of a $M_{\text {ZAMS }}=13 M_{\odot}$ progenitor and a delay time of less than one second ejects any silicon-layer material. The silicon-shell $Y_{\mathrm{e}}$ in this particular model 


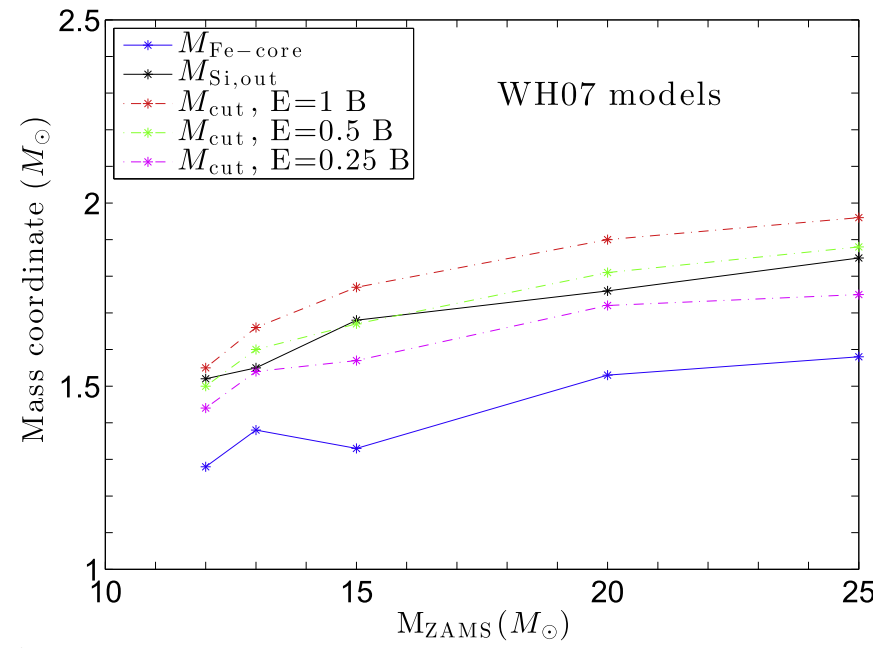

Figure 6. Position of $M_{\text {cut }}$ for different values of $E$, using the WH07 models.

$\left(Y_{\mathrm{e}}=0.491\right)$ is too low for SN 2012ec, but the value of $Y_{\mathrm{e}}$ in the silicon shell depends on details in the stellar evolution model. For larger $M_{\text {ZAMS }}$ and/or longer delay times, the mass cut lies above the outer edge of the silicon shell, so only oxygen-shell material with $Y_{\mathrm{e}}=0.499$ is ejected.

Figure 5 (bottom) shows the same quantities for the WH07 models. These models have slightly denser cores for a given $M_{\text {ZAMS }}$. The trends are, however, similar. The WH07 models show the same behavior of the outer edge of the silicon shell approaching the mass cut for low $M_{\mathrm{ZAMS}}$; in this grid they converge around $M_{\mathrm{ZAMS}}=12 M_{\odot}$. The mass cut lies just outside the silicon shell here rather than just inside as in the T96 models, but small changes in delay time and/or explosion energy will move the boundary.

Using either the T96 or WH07 model grids gives a consistent picture that ejecting the silicon layer at $M_{\text {ZAMS }}>13 M_{\odot}$ (and synthesizing $\left.M\left({ }^{56} \mathrm{Ni}\right) \sim 0.03 M_{\odot}\right)$ would require an explosion energy smaller than $1 \mathrm{~B}$. Figure 6 shows how $M_{\mathrm{Si} \text {-burn }}$ varies if $E$ is smaller than $1 \mathrm{~B}$. At $E=0.5 \mathrm{~B}$, ejection of silicon layers could occur up to $M_{\text {ZAMS }}=15 M_{\odot}$, but at larger $M_{\text {ZAMS }}$ still only oxygen layers are ejected. Going to $E=0.25$ B does allow for ejection of silicon layers at higher $M_{\text {ZAMS }}$. However, the expansion velocities scale as $V \propto \sqrt{E / M_{\text {ejecta }}}$, and for such a low explosion energy we would expect at least a factor 2 more narrow lines than usual. In SN 2012ec both photospheric and nebular lines appear as broad as in other SNe IIP (Barbarino et al. 2015, J15). This excludes the scenario of a low-energy explosion of a high-mass progenitor, which otherwise may be able to eject silicon-layer material (note that we have not adressed constraints from the dynamics here). An upper limit to the explosion energy for SN 2012ec can also be deduced; if $E$ was significantly greater than $1 \mathrm{~B}$ no progenitor could eject the silicon layer and still produce as little ${ }^{56} \mathrm{Ni}$ as $0.03 M_{\odot}$.

\subsection{Model Grids}

The next step is to see what $\mathrm{Ni} / \mathrm{Fe}$ ratios are produced in full explosion simulations. We let $M(\mathrm{Ni})$ and $M(\mathrm{Fe})$ denote the mass of all $\mathrm{Ni}$ and $\mathrm{Fe}$, respectively, one year after explosion. SN $2012 \mathrm{ec}$ has $M(\mathrm{Ni}) / M(\mathrm{Fe})=0.13-0.27 \quad$ and $M\left({ }^{56} \mathrm{Ni}\right)=0.02-0.04 M_{\odot}(\mathrm{J} 15)$. Figure 7 shows the measured position of $\mathrm{SN} 2012 \mathrm{ec}$ in the $M\left({ }^{56} \mathrm{Ni}\right)-M(\mathrm{Ni}) / M(\mathrm{Fe})$ plane

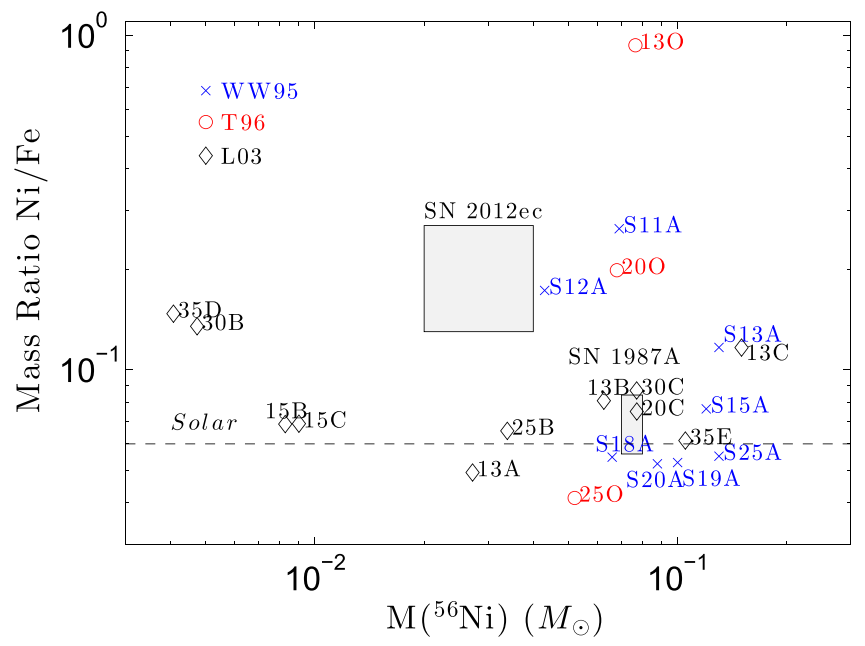

Figure 7. Position of $\mathrm{SN} 2012 \mathrm{ec} \quad\left(M\left({ }^{56} \mathrm{Ni}\right)=0.02-0.04 \quad M_{\odot}\right.$, $M(\mathrm{Ni}) / M(\mathrm{Fe})=0.13-0.27)$ in the $M\left({ }^{56} \mathrm{Ni}\right)-M(\mathrm{Ni}) / M(\mathrm{Fe})$ plane (shaded region), compared to the model grids presented by Woosley \& Weaver (1995, WW95), Thielemann et al. (1996, T96), and Limongi \& Chieffi (2003, L03). Also shown is the location of SN 1987A.

compared to the explosion simulations by Woosley \& Weaver (1995), T96, and Limongi \& Chieffi (2003).

The mass of ejected nickel in these models depends on the choice of mass cut/piston mass coordinate, which is set manually. Most of the models in the Woosley \& Weaver (1995) and T96 grids eject more ${ }^{56} \mathrm{Ni}$ than produced in SN 2012ec (so the mass cut is set "too deep"). They also produce a lower $\mathrm{Ni} / \mathrm{Fe}$ ratio. Some of the models in the Limongi \& Chieffi (2003) grid produce lower amounts of ${ }^{56} \mathrm{Ni}$, but none has the right combination of ${ }^{56} \mathrm{Ni}$ mass and $\mathrm{Ni} / \mathrm{Fe}$ ratio.

The T96 grid uses deeper mass cuts than is appropriate for SN 2012ec. For example, the mass cut used for their $20 M_{\odot}$. model is $1.61 M_{\odot}$, which adds $0.02 M_{\odot}$ of silicon-shell material with $\eta=0.494$ to the ejected ashes. This gives a dramatically higher $\mathrm{Ni} / \mathrm{Fe}$ ratio than if the mass cut were to have been placed at $1.67 M_{\odot}$, as needed for an ejected ${ }^{56} \mathrm{Ni}$ mass of $0.03 M_{\odot}$. For the $13 M_{\odot}$ model the $\mathrm{Ni} / \mathrm{Fe}$ value is 0.93 . This is burning of silicon-shell material with $Y_{\mathrm{e}}=0.491$. Comparing with Figure 1, the entropy must have been higher than what would have been needed to make a ratio consistent with SN 2012ec. Indeed, inspection of our MESA simulation shows that the progenitor density at the outer edge of the silicon shell is $\rho_{\text {pre }}=$ few $\times 10^{6} \mathrm{~g} \mathrm{~cm}^{-3}$ so the post-shock density (which is about seven times larger) is of order $\rho_{\text {post }}=10^{7} \mathrm{~g} \mathrm{~cm}^{-3}$. For $T \sim 5 \times 10^{9} \mathrm{~K}$ the entropies will be higher than needed for $Y_{\mathrm{e}}=0.490$ in Figure 1 . For the $25 M_{\odot}$ star, the $\mathrm{Ni} / \mathrm{Fe}$ ratio is 0.04 , too low for SN 2012ec. The burning occurred at lower entropy than needed for $Y_{\mathrm{e}}=0.499$.

The WW95 explosions use a piston rather than a thermal bomb. With this method, the choice of mass coordinate for the piston combined with a choice of explosion energy determines the mass cut (which equals the piston mass coordinate plus fallback). In the WW95 models the piston is placed at the edge of the iron core. Figure 8 shows the $\mathrm{Ni} / \mathrm{Fe}$ ratio in units of the solar value versus $M_{\text {ZAMS }}$ in the WW95 grid. The mass cut is inside the silicon shell for $M_{\text {ZAMS }} \lesssim 15 M_{\odot}$, raising the Ni/Fe production to several times the solar value. This is not offset by subsolar production at higher $M_{\text {ZAMS }}$; the burning occurs on the 


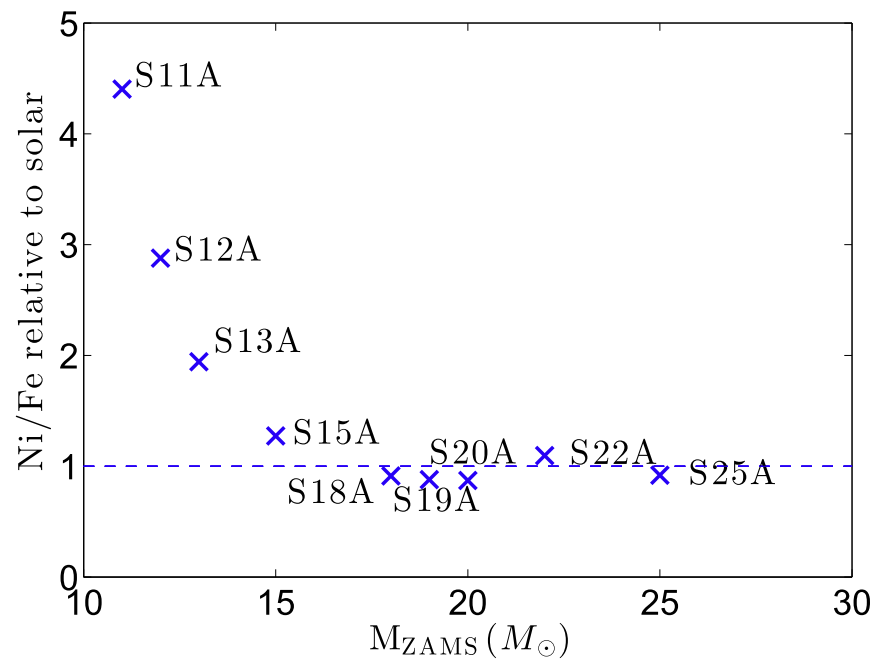

Figure 8. Ni/Fe ratio (relative to the solar value) in the WW95 model grid, for $E=1.2 \mathrm{~B}$ and solar metallicity.

large plateau region in Figure 1 . The $M_{\mathrm{ZAMS}}=11-13 M_{\odot}$ models give high enough $\mathrm{Ni} / \mathrm{Fe}$ to match $\mathrm{SN} 2012 \mathrm{ec}$, but the total mass of the ejected ashes is somewhat too large (Figure 7). The best fitting model is $12 \mathrm{~A}$, which makes $0.04 M_{\odot}$ of ${ }^{56} \mathrm{Ni}$ and gives a $\mathrm{Ni} / \mathrm{Fe}$ ratio of 0.18 , in close agreement with SN 2012ec.

The L03 models are piston-driven explosions, and nucleosynthesis yields for different piston motions at each $M_{\text {ZAMS }}$ are presented. Most models produce a $\mathrm{Ni} / \mathrm{Fe}$ ratio around solar. The only models with $\mathrm{Ni} / \mathrm{Fe}$ approaching SN 2012ec (30B and $35 \mathrm{D})$ only eject trace amounts of iron-group nuclei.

\section{DISCUSSION}

\subsection{Multidimensional Effects}

The ordered onion-like structure of iron-silicon-oxygen shells obtained in one-dimensional stellar evolution models may not be a good approximation of the true structure. Multidimensional simulations of the advanced stages of burning suggest strong convective overturns and a rearrangement of the various burning ashes (Bazán \& Arnett 1998; Meakin \& Arnett 2006; Arnett \& Meakin 2011; Meakin et al. 2011; Viallet et al. 2013; Couch et al. 2015). This opens up a possibility that neutron-rich material gets mixed out and resides at a larger mass coordinate than in 1D models, providing an alternative means of making a large $\mathrm{Ni} / \mathrm{Fe}$ ratio. Investigation of the explosive silicon burning process in such multidimensional progenitors would be of significant interest.

Also the explosion may involve asymmetries. Direct evidence for asymmetries in core-collapse explosions is available from imaging of Cas A (Fesen et al. 2006; Isensee et al. 2010; Hwang \& Laming 2012; Grefenstette et al. 2014) and SN 1987A (Wang et al. 2002; Kjær et al. 2010; Larsson et al. 2013; Boggs et al. 2015). If the explosion is asymmetric, the entropy for a fixed explosion energy becomes higher in the direction of the explosion, and the outer mass coordinate for complete silicon burning increases in this direction. At the same time, we expect deeper layers to be more easily ejected in the direction where the energy is focused. Thus, one expects the expelled silicon burning ashes to originate from a more extended mass range in the direction of the explosion.
Nagataki et al. (1997) studied the influence of asymmetries on the ${ }^{58} \mathrm{Ni} /{ }^{56} \mathrm{Ni}$ production using two-dimensional hydrodynamic simulations (see also Nagataki et al. 1998; Nagataki 2000). The study employed two different mass cuts: the first spherically symmetric, and the second sorting test particles by energy and making the mass cut at the energy giving the specified amount of ${ }^{56} \mathrm{Ni}$. For the second (more realistic) case, a higher degree of explosion asymmetry gave deeper mass cuts in the explosion direction, as expected. Silicon-layer material can thus more easily be ejected in the explosion direction, thereby achieving a high $\mathrm{Ni} / \mathrm{Fe}$ ratio.

Of significance for the analysis here is also their Case B model in which $Y_{\mathrm{e}}$ was artificially set to $Y_{\mathrm{e}}=0.499$ everywhere. This model illustrates the sole effect of a higher entropy achieved in asymmetric explosions $\left(S_{\gamma}\right.$ increases by a factor of 2-3 from the spherically symmetric case to their most asymmetric model). From Figure 1 we expect a quite modest change in $\mathrm{Ni} / \mathrm{Fe}$ for an entropy change of that order; indeed the ratio varies by less than $30 \%$ in the Nagataki et al. (1997) simulations. It is of interest to note that the ratio they obtained decreases with a higher degree of asymmetry. Considering Figure 1 again, this is likely because the burning region spans both the $\alpha$-rich freeze-out regime (where $\mathrm{Ni} / \mathrm{Fe}$ increases) and the $\alpha p$ regime (where $\mathrm{Ni} / \mathrm{Fe}$ decreases), and the net effect is a small decrease. This, in fact, adds further weight to the argument that the burning in SNe giving several times solar $\mathrm{Ni} /$ Fe ratios cannot have occured at as high $Y_{\mathrm{e}}$ as 0.499 .

\subsection{Neutrino-processed Ejecta}

Potentially, the material with high neutron excess could also come from the hot, neutrino-heated bubble deeper in the SN core (whose neutron-to-proton ratio has been reset by $n\left(\nu_{\mathrm{e}}, e^{-}\right) p, p\left(\overline{\nu_{\mathrm{e}}}, e^{+}\right) n$, and $p\left(e^{-}, \nu_{\mathrm{e}}\right) n$ reactions $)$ instead of being directly ejected after undergoing explosive burning. However, the similarity of $\nu_{\mathrm{e}}$ and $\bar{\nu}_{\mathrm{e}}$ luminosities and mean energies seen in modern simulations generally drives $Y_{\mathrm{e}}$ in the innermost ejecta above 0.5 (Fröhlich et al. 2006). Neutron-rich conditions can only be maintained in some of the early neutrino-heated ejecta if they expand rapidly enough, as in the electron capture SN model of Wanajo et al. (2011).

For $\mathrm{SNe}$ producing $M\left({ }^{56} \mathrm{Ni}\right) \gg 0.01 M_{\odot}$, this process does not provide a likely explanation, however. The total mass of iron-group material originating from the fast ejection of neutron-rich, neutrino-processed material is limited to a fraction of the mass of the gain region at shock revival in this scenario, i.e., to a few times $10^{-3} M_{\odot}$ (as in Wanajo et al. 2011). Moreover, the fast ejection of the early neutrinoheated ejecta in electron capture SNe depends crucially on the special density structure of their super-AGB progenitors, and it is doubtful whether sufficiently short expansion timescales could be reached in more massive progenitors.

\subsection{Nickel Isotopes in the Crab}

The Crab has a measured $\mathrm{Ni} / \mathrm{Fe}$ ratio of $60-75$ times solar (MacAlpine et al. 1989). This extreme ratio can be produced in the electron capture scenario described above, and indeed nucleosynthesis and expansion dynamics are consistent with such an origin (Nomoto et al. 1982; Kitaura et al. 2006; Wanajo et al. 2009). It is of interest to ask whether such a process is a unique solution, or whether explosive burning 
without any strong neutrino processing of the ejecta may also explain this value.

Inspection of Figure 1 shows that for $Y_{\mathrm{e}} \geqslant 0.495$ (the lowest value encountered outside the iron core), the only burning conditions that give such a $\mathrm{Ni} / \mathrm{Fe}$ ratio lie in the high entropy regime that is not matched by any physical shock speeds (Section 2.6). The most neutron-rich fuel $Y_{\mathrm{e}}=0.490$ can give such a $\mathrm{Ni} / \mathrm{Fe}$ ratio at lower and more typical explosion entropies $\left(\log \left(S_{\gamma} / R\right) \sim+0.5\right)$, but as discussed in Section 3.1, such a $Y_{\mathrm{e}}$ is too low for the silicon shell, and would be part of the iron core. It is difficult to achieve mass cuts that deep, and many other isotopes are produced in extremely non-solar proportions. It therefore appears a contrived scenario to explain the $\mathrm{Ni} / \mathrm{Fe}$ ratio in the $\mathrm{Crab}$ without the neutrino processing occurring in an electron capture event.

\subsection{Nickel Isotopes in SN 1987A}

In J15 it was demonstrated that, taking the optical depth of [Ni II] $6.636 \mu \mathrm{m}$ into account, the $\mathrm{Ni} / \mathrm{Fe}$ ratio in $\mathrm{SN} 1987 \mathrm{~A}$ derived from nebular-phase line luminosities is around solar, suggesting no or small ejection of silicon-shell material. This is consistent with the analysis in Kozma \& Fransson (1998), where a model with a nickel mass of 1.3 times solar relative to iron gave reasonable fits for [Ni II $6.636 \mu \mathrm{m}$ and $[\mathrm{Ni}$ II] $10.68 \mu \mathrm{m}$. These results supersede initial estimates of $\mathrm{Ni} / \mathrm{Fe} \lesssim 0.5$ times solar based on analytical formulae assuming optically thin emission (Rank et al. 1988; Wooden et al. 1993). The abundance of ${ }^{57} \mathrm{Ni}$ could be directly determined from gamma-ray lines, giving a ${ }^{57} \mathrm{Ni} /{ }^{56} \mathrm{Ni}$ ratio of $1-2$ times the solar ${ }^{57} \mathrm{Fe} /{ }^{56} \mathrm{Fe}$ ratio ${ }^{11}$ (Kurfess et al. 1992). A similar range was inferred from infrared lines (Varani et al. 1990) and light-curve models (Fransson \& Kozma 1993, 2002; Seitenzahl et al. 2014).

Figure 9 shows the production of $\mathrm{Ni} / \mathrm{Fe}$ (in units of solar) and ${ }^{57} \mathrm{Ni} /{ }^{56} \mathrm{Ni}$ (in units of solar ${ }^{57} \mathrm{Fe} /{ }^{56} \mathrm{Fe}$ ) for the $Y_{\mathrm{e}}=0.499$ case from the parameterized thermodynamic trajectories of Section 2. A Ni/Fe ratio of $0.75-1.5$ times solar and a ${ }^{57} \mathrm{Ni} /{ }^{56} \mathrm{Ni}$ ratio of 1-2 times solar are reproduced for burning at postshock densities of $\rho_{\mathrm{p}}=10^{6}-10^{8} \mathrm{~g} \mathrm{~cm}^{-3}$, which are typical values encountered in simulations (Figure 1). The revision of the $\mathrm{Ni} / \mathrm{Fe}$ ratio in $\mathrm{J} 15$ due to the calculated optical depths in the [Ni II] $6.636 \mu \mathrm{m}$ line is therefore important in allowing a consistent solution for the production of nickel isotopes in SN 1987A.

A lower $Y_{\mathrm{e}}$ moves the fit region to higher peak densities. For example, $Y_{\mathrm{e}}=0.497$ would require $\rho_{\mathrm{p}}>10^{8} \mathrm{~g} \mathrm{~cm}^{-3}$ to produce the SN 1987A values (not plotted, but see also Woosley \& Hoffman 1991). This density is larger than obtained in any published explosion models. Thus, the most consistent picture is that explosive burning in SN 1987A occurred in the oxygen shell, and that the mass cut therefore was outside the silicon shell. For a $M_{\text {ZAMS }}=20 M_{\odot}$ spherically symmetric progenitor model this is at $\sim 1.65 M_{\odot}$ in the T96 models and at $\sim 1.76 M_{\odot}$ in the WH07 models. From this we expect the minimum value of the mass cut to be around $1.7 M_{\odot}$. The baryonic neutron star mass was constrained to $1.2-1.7 M_{\odot}$ from the neutrino burst (Burrows 1988). This is marginally consistent with the constraints from the silicon burning, and disfavors a progenitor mass larger than $20 M_{\odot}$ (but allows for smaller).

\footnotetext{
${ }^{11}\left({ }^{57} \mathrm{Fe} /{ }^{56} \mathrm{Fe}\right) .=0.023$ (Lodders 2003).
}
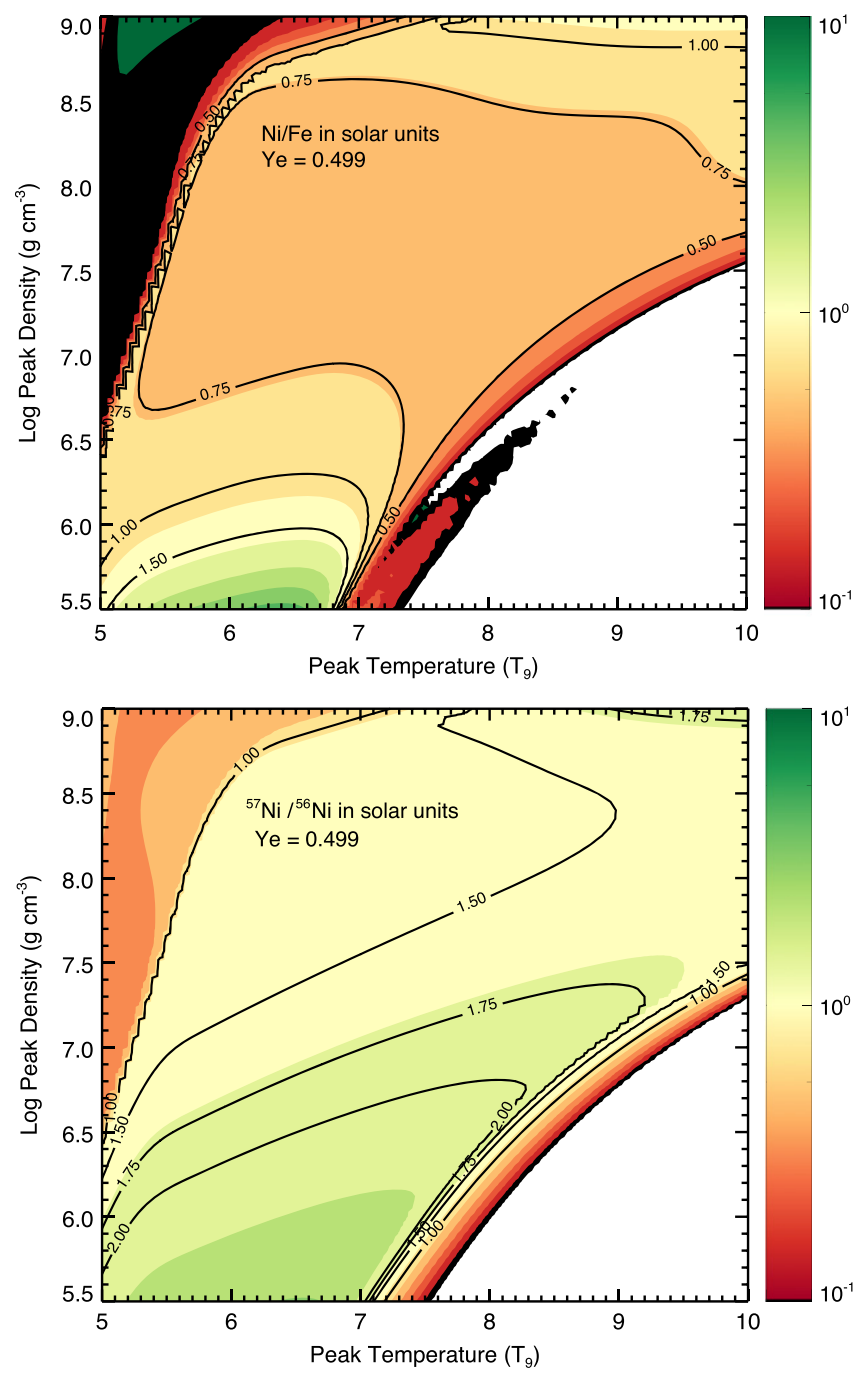

Figure 9. $\mathrm{Ni} / \mathrm{Fe}$ ratio in units of the solar ratio (top) and the ${ }^{57} \mathrm{Ni} /{ }^{56} \mathrm{Ni}$ ratio in units of the solar ${ }^{57} \mathrm{Fe} /{ }^{56} \mathrm{Fe}$ ratio (bottom), from the $Y_{\mathrm{e}}=0.499$ simulation.

\section{SUMMARY}

We have analyzed the production of nickel and iron (after radioactive decays) in explosive silicon burning, and the constraints on progenitor structure and burning conditions derivable from measured yields. We have focused on the implications of $\mathrm{Ni} / \mathrm{Fe}$ ratios of several times the solar value, as recently reported for the Type IIP SN 2012ec (J15), and previously for the SN Ic 2006aj (Maeda et al. 2007; Mazzali et al. 2007).

Using a nucleosynthesis network with parameterized thermodynamic trajectories, we have computed the nickel and iron yields (after decays) as a function of peak temperature, peak density, and neutron excess $\eta$ of the fuel. The $\mathrm{Ni} / \mathrm{Fe}$ ratio for most regimes is dominated by the production ratio of ${ }^{58} \mathrm{Ni} /$ $\left({ }^{54} \mathrm{Fe}+{ }^{56} \mathrm{Ni}\right)$. In the normal freeze-out regime, this ratio grows with increasing entropy as the storage of neutron excess switches from ${ }^{54} \mathrm{Fe}$ to ${ }^{58} \mathrm{Ni}$. When this switch is complete, the ratio reaches a plateau value of $\mathrm{Ni} / \mathrm{Fe}=\eta / 0.034$; fuels with $\eta=0.034 \times \mathrm{Ni} / \mathrm{Fe}$ provide a large region of allowed thermodynamic conditions. Smaller values of $\eta$ can also achieve the production, but only in a limited region of strong $\alpha$-rich freezeout where the ${ }^{58} \mathrm{Ni} /{ }^{56} \mathrm{Ni}$ ratio grows due to efficient depletion of ${ }^{56} \mathrm{Ni}$. We find that this process is not likely to be responsible for 
producing $\mathrm{Ni} / \mathrm{Fe}$ ratios several times solar as the necessary entropies would require unphysical shock speeds. Higher values of $\eta$ can achieve the production, but only in restricted regions of low entropy that are not encountered in typical explosion simulations. We conclude that to produce a $\mathrm{Ni} / \mathrm{Fe}$ ratio of a few times solar, burning at normal freeze-out or moderate $\alpha$-rich freeze-out (helium mass fraction $\left.X\left({ }^{4} \mathrm{He}\right) \lesssim 0.2\right)$ of a fuel with $\eta \sim 6 \times 10^{-3}\left(Y_{\mathrm{e}} \sim 0.497\right)$ is required.

The derived neutron excess value can be linked to the location in the star where explosive silicon burning occurred. Models for the progenitor structure show that the neutron excess successively increases inwards from the oxygen shell $\left(\eta \sim 2 \times 10^{-3}\right)$ to the iron core $\left(\eta \gtrsim 4 \times 10^{-2}\right)$. Only in the silicon shell is the neutron excess in the range needed for a $\mathrm{Ni} /$ Fe production of a few times the solar value $\left(\eta \sim 6 \times 10^{-3}\right)$. An exception is if the metallicity of the star exceeds five times solar, in which case the neutron excess in the oxygen shell increases to $\eta \sim 6 \times 10^{-3}$. Such a metallicity can be ruled out for $\mathrm{SN} 2012 \mathrm{ec}$ from $\mathrm{H}_{\mathrm{II}}$ region spectroscopy (Ramya et al. 2007). SN 2012ec, and other CCSNe producing large $\mathrm{Ni} / \mathrm{Fe}$ ratios, therefore likely burnt and ejected part of their silicon layers, which is a key constraint for explosion models.

In spherical symmetry, a given progenitor structure, explosion energy, delay time, and measured amount of ${ }^{56} \mathrm{Ni}$ define the position of the mass cut. Lower-mass stars have relatively thick silicon shells that more easily encompass the mass cut. We find that $M_{\text {ZAMS }} \lesssim 13 M_{\odot}$ progenitors exploding with a delay time of less than one second are able to eject part of their silicon layers. Such a progenitor for SN 2012ec is in agreement with the estimate of $M_{\text {ZAMS }}$ from modeling of the hydrostatic burning ashes $\left(M_{\text {ZAMS }}=13-15 M_{\odot}, \mathrm{J} 15\right)$. Higher-mass progenitors only eject oxygen-shell material with moderate $\alpha$-rich freeze-out, giving a $\mathrm{Ni} / \mathrm{Fe}$ ratio close to the solar value. In particular, the measured amounts of ${ }^{56} \mathrm{Ni},{ }^{57} \mathrm{Ni}$, and ${ }^{58} \mathrm{Ni}$ in SN 1987A appear consistent with the burning and ejection of pure oxygen-shell material. This in turn translates to a lower limit for the mass cut of this $\mathrm{SN}$ of $1.7 M_{\odot}$.

Asymmetry in the explosion can qualitatively change the $\mathrm{Ni}$ / Fe ratio by two means: by more easily ejecting deeper-lying silicon layers in the direction of the explosion, and by achieving a stronger $\alpha$-rich freeze-out. Analysis of published simulations (Nagataki et al. 1997) show that the entropy effect is insufficient, but the first mechanism can achieve a high $\mathrm{Ni} / \mathrm{Fe}$ ratio. An asymmetric explosion is a plausible explanation for the high Ni/Fe yield in SN 2006aj, an X-ray flash SN (Mazzali et al. 2006, 2007; Maeda et al. 2007).

For the Crab, a very high $\mathrm{Ni} / \mathrm{Fe}$ ratio of 60-75 times solar has been reported (MacAlpine et al. 1989). We find that this extreme value cannot be reproduced by any realistic entropy burning outside the iron core, and neutrino-neutronization obtained in electron capture models (Wanajo et al. 2009) remains the only viable explanation.

In conclusion, it is clear that constraints on both progenitor structures and explosion dynamics can be obtained from silicon-burning yields determined from nebular-phase spectra. The exact location of the silicon burning (i.e., the mass cut) has strong ramifications for iron-group yields used in galactic chemical evolution models, and the constraints derived from SN 2012ec and several other CCSNe provide important input for such modeling. Further observations and modeling of both core-collapse and thermonuclear supernovae in the nebular- phase, combined with explosive nucleosynthesis modeling, will enable us to make further progress in understanding SN explosion processes and the origin of the iron-group elements.

We thank K. Nomoto, F.-K. Thielemann, I. Seitenzahl, S. Rosswog, and R. Sethuram for discussion. The research leading to these results has received funding from the European Research Council under the European Union's Seventh Framework Programme (FP7/2007-2013)/ERC Grant agreement No[291222] (S.J.S.). S.J.S. acknowledges funding from STFC grants ST/I001123/1 and ST/L000709/1. F.X.T. was supported by NASA under TCAN grant NNX14AB53G, by NSF under SI $^{2}$ grant 1339600 , and by NSF under grant PHY1430152 for the Physics Frontier Center JINA. A.H. was supported by an ARC Future Fellowship (FT120100363) and JINA. B.M. acknowledges support by the Australian Research Council through a Discovery Early Career Research Award (DE150101145).

\section{REFERENCES}

Arnett, D. 1996, Supernovae and Nucleosynthesis: An Investigation of the History of Matter from the Big Bang to the Present (Princeton, NJ: Princeton Univ. Press)

Arnett, W. D. 1971, ApJ, 166, 153

Arnett, W. D., \& Meakin, C. 2011, ApJ, 733, 78

Arnett, W. D., \& Thielemann, F.-K. 1985, ApJ, 295, 589

Asplund, M., Grevesse, N., Sauval, A. J., \& Scott, P. 2009, ARA\&A, 47, 481 Barbarino, C., Dall'Ora, M., Botticella, M. T., et al. 2015, MNRAS, 448, 2312 Bazán, G., \& Arnett, D. 1998, ApJ, 496, 316

Bodansky, D., Clayton, D. D., \& Fowler, W. A. 1968, ApJS, 16, 299

Boggs, S. E., Harrison, F. A., Miyasaka, H., et al. 2015, Sci, 348, 670

Bresolin, F. 2007, ApJ, 656, 186

Bruenn, S. W., Mezzacappa, A., Hix, W. R., et al. 2013, ApJL, 767, L6

Burrows, A. 1988, ApJ, 334, 891

Chevalier, R. A. 1976, ApJ, 207, 872

Chieffi, A., \& Limongi, M. 2013, ApJ, 764, 21

Couch, R. C., \& Arnett, W. D. 1972, ApJ, 178, 771

Couch, S. M. 2013, ApJ, 775, 35

Couch, S. M., Chatzopoulos, E., Arnett, W. D., \& Timmes, F. X. 2015, arXiv: 1503.02199

Dessart, L., \& Hillier, D. J. 2011, MNRAS, 410, 1739

Fesen, R. A., Hammell, M. C., Morse, J., et al. 2006, ApJ, 645, 283

Fowler, W. A., \& Hoyle, F. 1964, ApJS, 9, 201

Fransson, C., \& Kozma, C. 1993, ApJL, 408, L25

Fransson, C., \& Kozma, C. 2002, NewAR, 46, 487

Fröhlich, C., Hauser, P., Liebendörfer, M., et al. 2006, ApJ, 637, 415

Grefenstette, B. W., Harrison, F. A., Boggs, S. E., et al. 2014, Natur, 506, 339

Hashimoto, M., Nomoto, K., \& Shigeyama, T. 1989, A\&A, 210, L5

Hix, W. R., \& Thielemann, F.-K. 1996, ApJ, 460, 869

Hix, W. R., \& Thielemann, F.-K. 1999, ApJ, 511, 862

Hwang, U., \& Laming, J. M. 2012, ApJ, 746, 130

Isensee, K., Rudnick, L., DeLaney, T., et al. 2010, ApJ, 725, 2059

Janka, H.-T., Hanke, F., Hüdepohl, L., et al. 2012, PTEP, 2012, 010000

Janka, H.-T., Müller, B., Kitaura, F. S., \& Buras, R. 2008, A\&A, 485, 199

Jerkstrand, A., Fransson, C., Maguire, K., et al. 2012, A\&A, 546, A28

Jerkstrand, A., Smartt, S. J., Fraser, M., et al. 2014, MNRAS, 439, 3694

Jerkstrand, A., Smartt, S. J., Sollerman, J., et al. 2015, MNRAS, 448, 2482

Kitaura, F. S., Janka, H.-T., \& Hillebrandt, W. 2006, A\&A, 450, 345

Kjær, K., Leibundgut, B., Fransson, C., Jerkstrand, A., \& Spyromilio, J. 2010, A\&A, 517, A51

Kozma, C., \& Fransson, C. 1998, ApJ, 497, 431

Kurfess, J. D., Johnson, W. N., Kinzer, R. L., et al. 1992, ApJL, 399, L137

Larsson, J., Fransson, C., Kjaer, K., et al. 2013, ApJ, 768, 89

Limongi, M., \& Chieffi, A. 2003, ApJ, 592, 404

Lodders, K. 2003, ApJ, 591, 1220

MacAlpine, G. M., McGaugh, S. S., Mazzarella, J. M., \& Uomoto, A. 1989, ApJ, 342, 364

Maeda, K., Kawabata, K., Tanaka, M., et al. 2007, ApJL, 658, L5

Magkotsios, G., Timmes, F. X., Hungerford, A. L., et al. 2010, ApJS, 191, 66

Magkotsios, G., Timmes, F. X., \& Wiescher, M. 2011, ApJ, 741, 78

Matzner, C. D., \& McKee, C. F. 1999, ApJ, 510, 379 
Maund, J. R., Fraser, M., Smartt, S. J., et al. 2013, MNRAS, 431, L102 Mazzali, P. A., Deng, J., Nomoto, K., et al. 2006, Natur, 442, 1018

Mazzali, P. A., Foley, R. J., Deng, J., et al. 2007, ApJ, 661, 892

Meakin, C. A., \& Arnett, D. 2006, ApJL, 637, L53

Meakin, C. A., Sukhbold, T., \& Arnett, W. D. 2011, Ap\&SS, 336, 123

Meyer, B. S., Krishnan, T. D., \& Clayton, D. D. 1998, ApJ, 498, 808

Müller, B., Janka, H.-T., \& Heger, A. 2012, ApJ, 761, 72

Nagataki, S. 2000, ApJS, 127, 141

Nagataki, S., Hashimoto, M.-a., Sato, K., \& Yamada, S. 1997, ApJ, 486, 1026

Nagataki, S., Hashimoto, M.-a., Sato, K., Yamada, S., \& Mochizuki, Y. S. 1998, ApJL, 492, L45

Nomoto, K., Sugimoto, D., Sparks, W. M., et al. 1982, Natur, 299, 803

Nordhaus, J., Burrows, A., Almgren, A., \& Bell, J. 2010, ApJ, 720, 694

Paxton, B., Bildsten, L., Dotter, A., et al. 2011, ApJS, 192, 3

Paxton, B., Cantiello, M., Arras, P., et al. 2013, ApJS, 208, 4

Perego, A., Hempel, M., Fröhlich, C., et al. 2015, arXiv:1501.02845

Ramya, S., Sahu, D. K., \& Prabhu, T. P. 2007, MNRAS, 381, 511

Rank, D. M., Pinto, P. A., Woosley, S. E., Bregman, J. D., \& Witteborn, F. C. 1988, Natur, 331, 505

Scheck, L., Kifonidis, K., Janka, H.-T., \& Müller, E. 2006, A\&A, 457, 963

Sedov, L. I. 1959, Similarity and Dimensional Methods in Mechanics (New York: Academic Press)

Seitenzahl, I. R., Timmes, F. X., \& Magkotsios, G. 2014, ApJ, 792, 10
The, L.-S., Clayton, D. D., Jin, L., \& Meyer, B. S. 1998, ApJ, 504, 500

Thielemann, F. K., \& Arnett, W. D. 1985, ApJ, 295, 604

Thielemann, F.-K., Hashimoto, M.-A., \& Nomoto, K. 1990, ApJ, 349, 222

Thielemann, F.-K., Nomoto, K., \& Hashimoto, M.-A. 1996, ApJ, 460, 408

Truran, J. W., Arnett, W. D., \& Cameron, A. G. W. 1967, CaJPh, 45, 2315

Truran, J. W., Cameron, A. G. W., \& Gilbert, A. 1966, CaJPh, 44, 563

Varani, G. F., Meikle, W. P. S., Spyromilio, J., \& Allen, D. A. 1990, MNRAS, 245,570

Viallet, M., Meakin, C., Arnett, D., \& Mocák, M. 2013, ApJ, 769, 1

Wanajo, S., Janka, H.-T., \& Müller, B. 2011, ApJL, 726, L15

Wanajo, S., Nomoto, K., Janka, H.-T., Kitaura, F. S., \& Müller, B. 2009, ApJ, 695,208

Wang, L., Wheeler, J. C., Höflich, P., et al. 2002, ApJ, 579, 671

Wongwathanarat, A., Janka, H.-T., \& Müller, E. 2010, ApJL, 725, L106

Wooden, D. H., Rank, D. M., Bregman, J. D., et al. 1993, ApJS, 88, 477

Woosley, S. E. 1988, ApJ, 330, 218

Woosley, S. E., Arnett, W. D., \& Clayton, D. D. 1973, ApJS, 26, 231

Woosley, S. E., \& Heger, A. 2007, PhR, 442, 269

Woosley, S. E., Heger, A., \& Weaver, T. A. 2002, RvMP, 74, 1015

Woosley, S. E., \& Hoffman, R. D. 1991, ApJL, 368, L31

Woosley, S. E., \& Hoffman, R. D. 1992, ApJ, 395, 202

Woosley, S. E., \& Weaver, T. A. 1995, ApJS, 101, 181

Young, P. A., \& Fryer, C. L. 2007, ApJ, 664, 1033 